This item was submitted to Loughborough's Research Repository by the author.

Items in Figshare are protected by copyright, with all rights reserved, unless otherwise indicated.

\title{
Investigation of bovine serum albumin denaturation using ultrasonic spectroscopy
}

PLEASE CITE THE PUBLISHED VERSION

http://dx.doi.org/10.1016/j.foodhyd.2010.11.011

PUBLISHER

(C) Elsevier

VERSION

AM (Accepted Manuscript)

LICENCE

CC BY-NC-ND 4.0

\section{REPOSITORY RECORD}

Povey, Malcolm J.W., Jonathan D. Moore, Julian Braybrook, Howard Simons, Ron Belchamber, Meera Raganathan, and Valerie J. Pinfield. 2012. "Investigation of Bovine Serum Albumin Denaturation Using Ultrasonic Spectroscopy”. figshare. https://hdl.handle.net/2134/11088. 
This item was submitted to Loughborough's Institutional Repository (https://dspace.lboro.ac.uk/) by the author and is made available under the following Creative Commons Licence conditions.

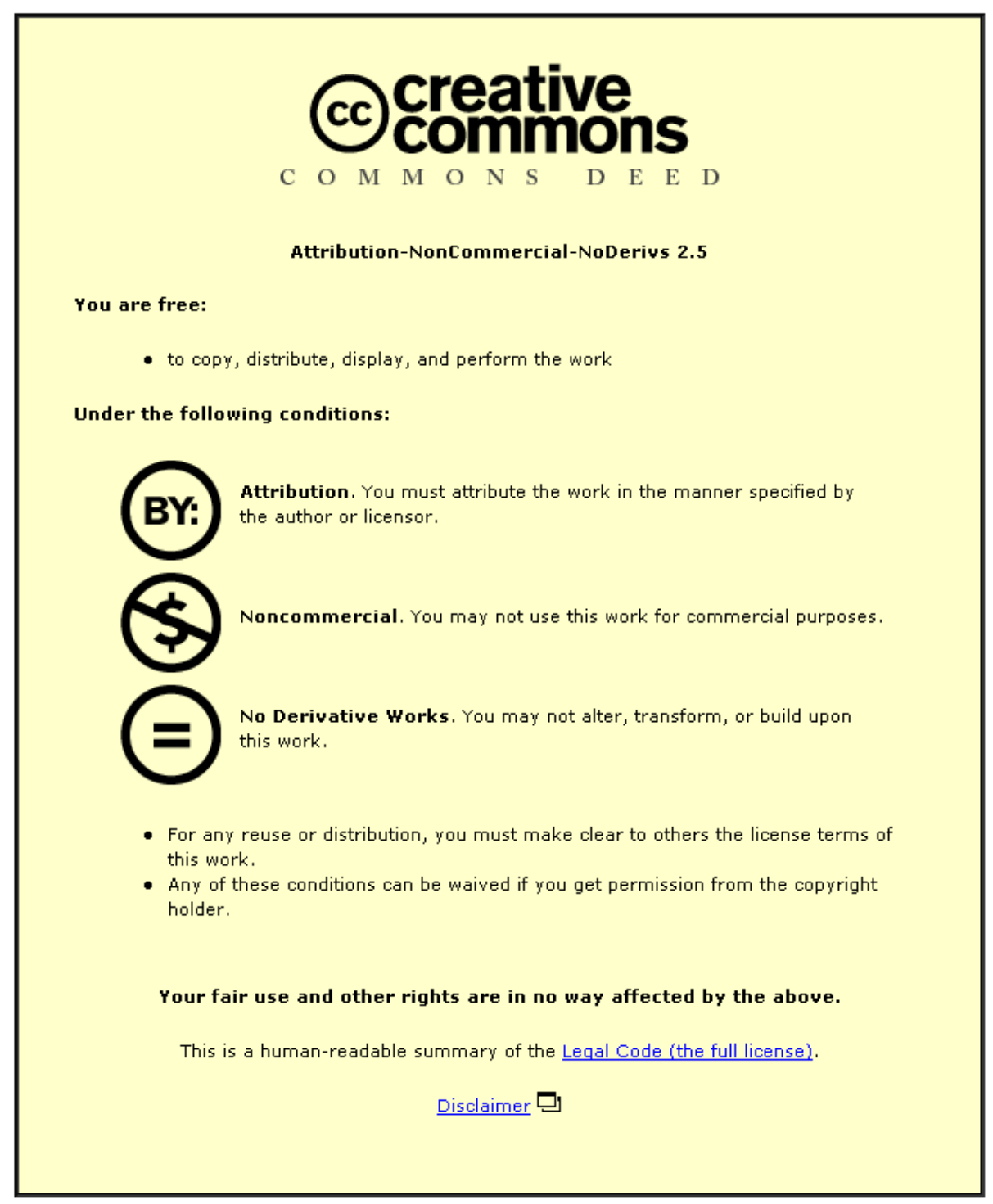

For the full text of this licence, please go to: http://creativecommons.org/licenses/by-nc-nd/2.5/ 
Elsevier Editorial System(tm) for Food Hydrocolloids

Manuscript Draft

Manuscript Number: FOODHYD-D-10-00198R1

Title: Investigation of bovine serum albumin denaturation using ultrasonic spectroscopy

Article Type: Original Research Paper

Keywords: ultrasound spectroscopy; compressibility; bovine serum albumin; aggregation; gelation; protein-protein interactions; protein-solvent interactions.

Corresponding Author: Professor Malcolm JW Povey,

Corresponding Author's Institution: University of Leeds

First Author: Malcolm J Povey, School of Food Science and Nutrition

Order of Authors: Malcolm J Povey, School of Food Science and Nutrition; Malcolm JW Povey; Jonathan D Moore, PhD; Julian Braybrook, PhD; Howard Simons; Ron Belchamber, PhD; Valerie Pinfield, PhD; Meera Raganathan, PhD; Malcolm J Povey, PhD, FInstP, CEng,CPhys; Jonathan D Moore, PhD; Julian Braybrook, PhD; Howard Simons; Ron Belchamber; Meera Ranganathan; Valerie Pinfield, PhD

Abstract: The ability of ultrasound spectroscopy to characterise protein denaturation at relatively high concentrations and under conditions found in foods, is examined. Measurement of longitudinal sound velocity against concentration and frequency $(20-160 \mathrm{MHz})$ for the bovine serum albumin monomer at $\mathrm{pH} 7.0$ gave a frequency independent value for molecular compressibility of at $25^{\circ} \mathrm{C}$, corresponding to a sound velocity for the BSA molecule of $1920 \mathrm{~ms}-1$. At $160 \mathrm{MHz}$, the longitudinal sound attenuation in BSA molecules is $\sim 5200 \mathrm{Npm}-1$, a factor of 10 higher than in water. The excess attenuation of the solution over water was nearly $90 \mathrm{Npm}-1$ at the highest measured volume fraction of 0.03 (or $3 \% \mathrm{v} / \mathrm{v}$ ). Concentration-dependent ultrasound velocity (20 - $160 \mathrm{MHz})$ and attenuation $(2-120 \mathrm{MHz})$ spectra were obtained over time for heated bovine serum albumin (BSA) solutions up to $40 \mathrm{mg} / \mathrm{mL}$ at neutral $\mathrm{pH}$ and at $25^{\circ} \mathrm{C}$. An acoustic scattering model was used which considered the solute molecules as scatterers of ultrasound, to determine the molecules' sound velocity, compressibility, and attenuation properties. Mild heat treatment caused the molecule to organise into dimers and trimers, without change in sound velocity; implying that there is little or no change in secondary structure. Changes in attenuation spectra correlated with estimated molecular weight as determined through DLS and SEC measurements. During oligomerisation, the BSA molecules continue to behave acoustically as monomers.

Under severe heat treatment, BSA rapidly suffered irreversible denaturation and gelation occurred which affected both ultrasound attenuation spectra and the velocity of sound, consistent with significant molecular conformation changes and/or molecule-molecule interactions. 



Ultrasound spectroscopy (velocity and attenuation 2-160 MHz) measurements can follow all stages in the denaturation of bovine serum albumin, from dimerisation through to severe denaturation. Velocity and attenuation relate to different features of the molecule. Velocity is sensitive to changes in conformation, secondary structure, and any significant changes in elastic or shear properties, attenuation is affected by relaxation processes and possibly aggregation. 
For Food Hydrocolloids: Investigation of bovine serum albumin denaturation

\section{$1 \quad$ Investigation of bovine serum albumin denaturation using}

\section{ultrasonic spectroscopy}

3 Malcolm J. W. Povey ${ }^{\mathrm{a}^{*}}$, Jonathan D. Moore ${ }^{\mathrm{b}}$, Julian Braybrook ${ }^{\mathrm{c}}$, Howard Simons ${ }^{\mathrm{c}}$, Ron

4 Belchamber $^{\mathrm{d}}$, Meera Raganathan ${ }^{\mathrm{e}}$, Valerie Pinfield ${ }^{\mathrm{f}}$

$5 *$ Corresponding author : Malcolm Povey, Phone +44 1133432963 Fax +44 1133432982, E-

$6 \quad$ mail: m.j.w.povey@leeds.ac.uk,

$7 \quad{ }^{a}$ School of Food Science and Nutrition, University of Leeds, Leeds LS2 9JT

$8 \quad$ b National Physical Laboratory, Hampton Road, Teddington, Middlesex, TW11 0LW

$9 \quad{ }^{\mathrm{c}}$ LGC, Queens Road, Teddington, Middlesex TW11 0LY

$10{ }^{\mathrm{d}}$ Process Analysis and Automation Ltd, Fernhill Road, Farnborough, Hampshire, GU14 9RX

$11{ }^{\mathrm{e}}$ Department of Systems Engineering, Brunel University, Uxbridge, Middlesex UB8 3PH

$12{ }^{\mathrm{f}}$ Electrical Systems and Optics Division, Faculty of Engineering, University of Nottingham,

13 Nottingham, NG7 2RD

14

The ability of ultrasound spectroscopy to characterise protein denaturation at relatively high

17 concentrations and under conditions found in foods, is examined. Measurement of

18 longitudinal sound velocity against concentration and frequency (20-160 MHz) for the bovine

19 serum albumin monomer at $\mathrm{pH} 7.0$ gave a frequency independent value for molecular

20 compressibility of $\kappa^{\prime}=2.05 \times 10^{-10} \mathrm{~Pa}^{-1}$ at $25{ }^{\circ} \mathrm{C}$, corresponding to a sound velocity for the

21 BSA molecule of $1920 \mathrm{~ms}^{-1}$. At $160 \mathrm{MHz}$, the longitudinal sound attenuation in BSA Page 1 of 29, printed 14/11/201016:04:35 
For Food Hydrocolloids: Investigation of bovine serum albumin denaturation

22 molecules is $\sim 5200 \mathrm{Npm}^{-1}$, a factor of 10 higher than in water. The excess attenuation of the

23 solution over water was nearly $90 \mathrm{Npm}^{-1}$ at the highest measured volume fraction of 0.03 (or

$243 \% \mathrm{v} / \mathrm{v})$. Concentration-dependent ultrasound velocity $(20-160 \mathrm{MHz})$ and attenuation (2 $120 \mathrm{MHz}$ ) spectra were obtained over time for heated bovine serum albumin (BSA) solutions up to $40 \mathrm{mg} / \mathrm{mL}$ at neutral $\mathrm{pH}$ and at $25{ }^{\circ} \mathrm{C}$. An acoustic scattering model was used which considered the solute molecules as scatterers of ultrasound, to determine the molecules' sound velocity, compressibility, and attenuation properties. Mild heat treatment caused the molecule to organise into dimers and trimers, without change in sound velocity; implying that there is little or no change in secondary structure. Changes in attenuation spectra correlated with estimated molecular weight as determined through DLS and SEC measurements. During oligomerisation, the BSA molecules continue to behave acoustically as monomers.

Under severe heat treatment, BSA rapidly suffered irreversible denaturation and gelation occurred which affected both ultrasound attenuation spectra and the velocity of sound, consistent with significant molecular conformation changes and/or molecule-molecule interactions.

Keywords: ultrasound spectroscopy; compressibility; bovine serum albumin; aggregation; gelation; protein-protein interactions; protein-solvent interactions.

\section{1. Introduction}

40 Ultrasound spectroscopy is increasingly used to investigate the properties of biomolecules,

41 for example functional properties such as storage modulus arising from protein-protein 42 interactions in high protein concentration solutions (Saluja, Badkar, Zeng, Nema, \& Kalonia, 43 2007) and for folding studies (El Kadi, et al., 2006). In this work we measure for the first 44 time both velocity spectra $(20-160 \mathrm{MHz})$ and attenuation spectra $(2-160 \mathrm{MHz})$. Velocity Page 2 of 29, printed 14/11/201016:04:35 
For Food Hydrocolloids: Investigation of bovine serum albumin denaturation

and attenuation relate to different features of the molecule. Velocity is sensitive to changes in conformation, secondary structure, and any significant changes in elastic or shear properties, attenuation is affected by relaxation processes and possibly aggregation. Ultrasound velocity has the unique property that it is affected by the adiabatic compressibility of the molecule and hence is sensitive to molecular transitions that alter compressibility. An example of such a transition is crystallization, to which ultrasound velocity is very sensitive, primarily due to adiabatic compressibility (or bulk modulus) change associated with the phase change (Povey, Awad, Huo, \& Ding, 2007). Ultrasound has the further advantage that it can be used for bulk measurement in optically opaque, concentrated or multiple scattering media and is therefore a complementary technique to light scattering and circular dichroism. Unlike circular dichroism however, ultrasound does not provide specific information about secondary and tertiary structure, but does respond to changes in molar free energy (Chalikian, 2008). The key advantage of ultrasound in this work is its ability to provide information at all stages in the aggregation of the protein BSA, from dimerisation through to gelation, offering a method for characterising proteins under the conditions in which they are used in foods.

In this work we use a method previously reported in this journal (Apenten, Buttner, Mignot, Pascal, \& Povey, 2000) for obtaining molecular compressibility of BSA from ultrasound measurements of velocity and attenuation. Our previous study was carried out at a single frequency and only velocity was measured. As in the previous study, here it is assumed that the protein molecule is a particle that scatters ultrasound according to classical scattering theory.

A number of mechanisms, other than that of scattering, have been proposed for the features in the absorption spectra of protein solutions including BSA (Kessler \& Dunn, 1969; Hussey \& Edmonds, 1971; Holmes \& Challis, 1996). These include changes in conformation, proton 
For Food Hydrocolloids: Investigation of bovine serum albumin denaturation

transfer reactions, solvation effects and other relaxation effects. Models have been developed to estimate the contribution of the proposed mechanisms to the attenuation. In particular, relaxation models are used to identify the relaxation frequencies (Holmes \& Challis, 1996). Some workers have fitted a small number of relaxations in their experimental frequency range, whilst others fit a continuous range of relaxation frequencies.

(Bryant \& McClements, 1999) proposed that the overall attenuation of ultrasound by a protein solution $\left(\alpha_{\text {soln }}\right)$ be divided into molecular relaxation $\left(\alpha_{\mathrm{MR}}\right)$ and scattering contributions $\left(\alpha_{\mathrm{s}}\right)$. This presupposes that the relaxation contribution can be identified prior to separating out the scattering contribution. Holmes and Challis (1996) found that at a temperature of $37^{\circ} \mathrm{C}$, higher than that used in this study, a large frequency squared dependent term needed to be subtracted from their data, before a two frequency relaxation model could be fitted to their data. Whilst they suggest this subtracted term is due to another high frequency relaxation, our data suggest that the subtracted term could be, at least in part, due to scattering of sound by the BSA molecule. In this paper we analyse the attenuation data entirely in the context of the scattering model, without attempting to separate the relaxation contribution.

\subsection{Scattering model}

The straightforward classical model of sound propagation relates the velocity of sound $v$ to the elastic modulus $M$ and density of a material $\rho$ through

$v=\sqrt{M / \rho}$

The elastic modulus depends on the type of wave propagating and the material through which it propagates. In this work we measure only the longitudinal propagation mode (also called the compressional mode) which is a pressure wave. Other modes include shear and surface modes. The shear mode requires a significant shear modulus, $G$, in order to propagate. In Page 4 of 29, printed 14/11/201016:04:35 
For Food Hydrocolloids: Investigation of bovine serum albumin denaturation

92 solid materials the elastic modulus governing longitudinal wave propagation is related to the

93 bulk modulus $K_{B}$ given by

$94 \quad M=K_{B}+4 G / 3$

In fluids such as the protein solutions which form most of the subject of this paper, the elastic modulus is given by the bulk modulus which is related to the adiabatic compressibility $\kappa$

97 through

$98 M=K_{B}=1 / \kappa$

99 The classical model is formally correct but cannot predict the effects of scattering by small

100 particles. In contrast, the scattering model approach considers each molecule or dispersed

101 phase particle as a scattering object, which has the effect of removing energy from the

102 forward transmitted sound wave (usually an incident plane wave) and scattering it in all

103 directions (Povey, 1997). Such scattering may affect both longitudinal wave attenuation

104 (through the wave amplitude) and velocity (through a change in phase of the wave). These

105 ultrasound propagation parameters depend on the properties of the continuous and dispersed 106 phase materials, and the concentration of the dispersed matter (Challis, Povey, Mather, \&

107 Holmes, 2005; Povey, 1997).

108 In the present work, the systems were measured in the long wavelength region, in which the 109 wavelength of the sound wave is much larger than the scatterer (molecule) radius. In this 110 case, the scattering model shows that the wave number $\left(K_{\text {soln }}\right.$, not to be confused with the 111 bulk modulus $K_{\mathrm{B}}$ ) of the solution can be written in terms of the solvent (water) wave number

112 ( $K=\frac{\omega}{v}+i \alpha$, where $\omega$ is radial frequency, $v$ is sound velocity, $i=\sqrt{-1}$ and $\alpha$ is attenuation 113 coefficient). At the concentrations used in this study, the second order (in concentration) 114 multiple scattering term, is negligible, and the attenuation and velocity vary linearly with the Page 5 of 29, printed 14/11/201016:04:35 
For Food Hydrocolloids: Investigation of bovine serum albumin denaturation

115 concentration $(\varnothing)$ expressed as the product of partial molar volume (Table 1 and Table 2) and

116 the mole fraction as a fraction of the mean molar volume of the solution (Povey, 1997):

$117\left(\alpha_{\text {soln }}-\alpha_{\text {solv }}-\alpha_{\mathrm{MR}}\right)=C_{\alpha} \varnothing+O\left(\varnothing^{2}\right)$

$1181 / v_{\text {soln }}^{2}-1 / v_{\text {solv }}^{2}=C_{v} \emptyset+O\left(\emptyset^{2}\right)$

119 Here subscripts soln and solv refer to the properties of the protein solution and to the solvent

120 containing buffer. The scattering model shows that the concentration dependence is obtained

121 as a volume fraction, rather than other concentration measures often used such as weight

122 fraction. This means that the partial molar volume (Table 1) has a significant impact on sound

123 velocity. Attenuation and inverse square velocity show a linear dependence in volume

124 fraction when measured relative to the same parameter in the pure solvent. The parameters

$125 C_{\alpha}$ and $C_{v}$ define the respective gradients of the excess parameters with volume fraction.

126 Note in relation to those data analyses that extrapolate linearly to infinite dilution, scattering

127 theory predicts the inverse square velocity of sound to depend linearly on volume

128 concentration (Equation 5).

129 According to the scattering model, the gradient $C_{v}$ obtained from the velocity measurements

130 relates to the real parts of the coefficients given above, which are a fractional compressibility

131 difference $\Delta \kappa / \kappa$, a fractional density difference $\Delta \rho / \rho$ and a thermal contribution $Y$.

$C_{v}=\left(1 / v^{2}\right)\{(\Delta \kappa / \kappa)+(\Delta \rho / \rho)+\mathrm{Y}\}$

133 Each of the terms is independent of frequency in the long wavelength region. Thus it is

134 expected that the velocity gradient parameter should be independent of frequency. For the

135 thermal term, the frequency independence only applies when the thermal wavelength is also 
For Food Hydrocolloids: Investigation of bovine serum albumin denaturation

136 long, so that if the velocity gradient is indeed independent of frequency, the validity of this

137 limit is confirmed. Rearranging the expression leads to the compressibility of the molecules.

$138 \quad \kappa^{\prime}=\kappa\left[1+v^{2} C_{v}-(\Delta \rho / \rho)-Y\right]$

139 where the factor $Y$ arises from thermal scattering (Povey, 1997).

140 The molar compressibility and hence the sound velocity will be affected by changes in partial

141 molar volume, heat capacity, solvation and the pressure of the applied acoustic field.

142 Because the acoustic field has frequency and duration, relaxation effects will certainly be

143 present. The pressure may have an effect because an acoustic field is a pressure fluctuation

144 with amplitude of approximately $20 \mathrm{kPa}$ for a typical $100 \mathrm{~W} \mathrm{~m}{ }^{-2}, 1 \mathrm{MHz}$ pulse in water at

$14525{ }^{\circ} \mathrm{C}$ (Povey \& McClements, 1988; Povey, 1997). No attempt has been made in this work to

146 unravel in detail the relative contributions of these effects during oligomerisation and

147 denaturation of the studied protein, although we studied the frequency dependence of both

148 sound velocity and attenuation. Determined compressibility includes both intrinsic and

149 hydration terms.

\section{Materials and Methods}

\subsection{Protein solutions}

152 The water used in all experiments is MilliQ water (Millipore, Ma, USA), prepared by ion exchange, used because it gives much more reproducible results than triple distilled water in ultrasound experiments.

155 Bovine serum albumin (BSA- Product number A2153, Fraction V, Sigma-Aldrich Company

156 Ltd, Gillingham, UK) is a widely used globular protein with a molecular weight of $66500 \mathrm{Da}$

157 (Murayama \& Tomida, 2004). $40 \mathrm{~g} / \mathrm{l}$ solutions of BSA were prepared by the slow dissolution Page 7 of 29, printed 14/11/201016:04:35 
For Food Hydrocolloids: Investigation of bovine serum albumin denaturation

158 of the freeze-dried protein in $0.1 \mathrm{M}$ potassium phosphate buffer $(\mathrm{pH} \mathrm{9.6)}$ and then stored at

$1594{ }^{\circ} \mathrm{C}$, prior to use. Just before measurement, samples were buffered at $\mathrm{pH}$ 7.0.

160 Concentrations are quoted as mass of protein in volume of solution and later converted to 161 volume fraction by using the solution density measurements. Solutions were degassed in an

162 ultrasonic bath. For the cross platform and oligomerisation studies $5 \mathrm{~g} / \mathrm{l}$ solutions of BSA 163 were finally prepared in $20 \mathrm{mM}$ potassium phosphate buffered to $\mathrm{pH}$ 7.0.

\section{$164 \quad 2.2$ Oligomerisation experiments}

165 Samples were prepared in $10 \mathrm{ml}$ aliquots and heated in a water bath at $70{ }^{\circ} \mathrm{C}+/-0.02{ }^{\circ} \mathrm{C}$ for 16660,120 , and $180 \mathrm{~min}$. After heating, the samples were allowed to cool to room temperature.

167 The control sample was maintained at room temperature. Sub-samples were removed after 168 heating and were analysed by dynamic light scattering (DLS) and size exclusion 169 chromatography (SEC). The remaining sample was analysed by ultrasound spectrometry.

\subsection{Gelation experiments}

171 The BSA solutions were heated in a thermostat-controlled oil-bath (refrigerated and heating circulator from Jubalo, F25-MC, accuracy $+/-0.02{ }^{\circ} \mathrm{C}$ ) so that the time-temperature profiles could be controlled and registered. The solutions were sealed in A4 cans and maintained at $25{ }^{\circ} \mathrm{C}$ during $10 \mathrm{~min}$ for the equilibrium time. They were then heated up to $80{ }^{\circ} \mathrm{C}$ over a period of 25 min, maintained at $80{ }^{\circ} \mathrm{C}$ during a few minutes $(0,1,2,3,4,5,6,7,8,9,10,11$, $12,13,14,15$ or 16 minutes), and finally cooled down to $25{ }^{\circ} \mathrm{C}$ during 25 minutes. In-can temperature was measured in two positions and logged together with the bath temperature using type $\mathrm{T}$ thermocouples. Measurements of the storage modulus $\left(G^{\prime}\right)$ and of the loss modulus $\left(G^{\prime \prime}\right)$ were performed using a Bohlin C-VOR rheometer from Bohlin Instruments. 
For Food Hydrocolloids: Investigation of bovine serum albumin denaturation

180 The measuring system used was C14, cup and vane tool (vane diameter of $14 \mathrm{~mm}$ and gap

181 width of $0.15 \mathrm{~mm}$ ), which has a stress range of $1-750 \mathrm{~Pa}$, with a strain sensitivity of $\sim 10^{-4}$.

182 After pre-shearing, 4 drops of silicone oil AS4 (Fluka, from Sigma-Aldrich) were added to

183 avoid evaporation. The temperature was set to $80^{\circ} \mathrm{C}$, and $G^{\prime}$ and $G^{\prime \prime}$ were measured during

1842 hours. More severe heat treatments were applied using a computer controlled steam retort

185 (Figure 1).

$186 \quad 2.4$ Instrumentation

\section{$187 \quad 2.4 .1$ Ultrasound velocity and attenuation}

188 Ultrasound velocity and attenuation measurements were made using the ICHOS II instrument

189 (PAA Ltd, Farnborough, UK). The system is based on the "pitch and catch" method, with a

190 longitudinal transmitted pulse passed through the sample to a receiver; in the frequency range

191 1-200 MHz, operating in the near field. In practice, this operating frequency range is

192 significantly reduced by signal to noise considerations. The sample cell is made of stainless

193 steel and requires a sample volume of 7-11 ml, with a path length for the ultrasound signal of

$19411.2 \mathrm{~mm}$ at $25^{\circ} \mathrm{C}$. The cell temperature is maintained by a water jacket and homogeneity is

195 assisted by a magnetic stirrer. The sample temperature in all experiments was

$19625^{\circ} \mathrm{C} \pm 0.2^{\circ} \mathrm{C}$.

197 The instrument measures the intensity of the ultrasound signal received after transmission

198 through the sample, and the time of flight of that signal in the sample. For instrument details,

199 please refer to the manufacturer's documentation. Measurements are calibrated against a

200 reference sample, in this case, pure water because of the high accuracy data available. The

201 velocity of longitudinal sound in pure water is obtained by the "148-point" equation of

202 Bilaniuk \& Wong, (1993, 1996), which defines its temperature dependence up to fifth order. Page 9 of 29, printed 14/11/201016:04:35 
For Food Hydrocolloids: Investigation of bovine serum albumin denaturation

203 The measured temperature was $24.8^{\circ} \mathrm{C}$, and the velocity in water is therefore $1496.2 \mathrm{~ms}^{-1}$ 204 and independent of frequency.

205 The velocity in the sample is determined by calibrating the time of flight against that 206 measured in pure water at each frequency. Similarly, for attenuation, the intensity measured 207 in the sample is calibrated against that measured in water.

208 The measurements were found to be accurate over the range 20-160 MHz. Outside this 209 range, the intensity in water (and sample) was too low. Velocity measurements were carried

210 out in the ICHOS II spectrometer with an accuracy of $\pm 0.2 \mathrm{~m} \mathrm{~s}^{-1}$. The main determinant of 211 the accuracy of ultrasound velocity measurements is temperature control $( \pm 200 \mathrm{mK})$ because

212 the temperature coefficient of the velocity of sound in water at ambient temperature and

213 pressure is $3 \mathrm{~ms}^{-10} \mathrm{C}^{-1}$. Relative to velocity, ultrasound attenuation is much less sensitive to 214 temperature variation.

215 Data were also obtained using a Malvern Ultrasizer ultrasound spectrometer. This operates

216 by producing a quasi-static longitudinal standing wave in a chamber holding at least $500 \mathrm{ml}$ 217 of sample, within which transducers, operating between $2 \mathrm{MHz}$ and $120 \mathrm{MHz}$, move. The 218 transducers are moved to obtain optimal signal to noise ratio and hence accuracy, within 219 given frequency bands. This contrasts with the PAA instrument whose transducers operate at 220 a fixed path length and which operate in pulsed mode. The Ultrasizer produces absolute 221 attenuation spectra, whereas the PAA system operates in a comparative mode with water.

\section{$222 \quad$ 2.4.2 Dynamic light scattering}

223 A Malvern Zetasizer Nano was used at both NPL and Leeds to follow the size of the BSA 224 and its aggregated states. There are some issues with interpretation of data for the highly 225 aggregated forms of BSA as the light scattering technique relies on particles undergoing Page 10 of 29, printed 14/11/201016:04:35 
For Food Hydrocolloids: Investigation of bovine serum albumin denaturation

226 Brownian motion. In particular, once a gel network forms in which diffusion is arrested,

227 sizing can no longer be carried out. The refractive index used for the BSA is 1.45 (the protein

228 refractive index) and a value of 0.00 for the absorption (at the laser wavelength of $633 \mathrm{~nm}$ ).

229 For the phosphate buffer (the dispersant), the viscosity measured was $1.04 \mathrm{cP}$ with 1.3357 for

230 the refractive index.

\subsubsection{Density and partial molar volume}

232 The method to obtain the volume fraction for the ultrasound measurements was as described 233 in Apenten et al. (2000) with partial molar volume determined according to the method of

234 Atkins (Atkins \& de Paula, 2002). Measurements of density (Table 1) were made using a 235 vibrating tube densitometer (Anton Paar, Gratz, Austria) for solutions of various 236 concentrations.

\section{$237 \quad$ 2.4.2 Size Exclusion Chromatography}

238 For all SEC measurements the HPLC (Jasco) was operated using Borwin (Version 1) software. Control and heat-treated BSA measurement samples were injected (10 $\mu 1)$ onto a BioSep-SEC-S2000 (Phenomenex) column equilibrated in $1 \mathrm{x}$ PBS [10 mM phosphate

241 buffer, $2.7 \mathrm{mM} \mathrm{KCl}, 137 \mathrm{mM} \mathrm{NaCl}, \mathrm{pH} \mathrm{7.4]} \mathrm{running} \mathrm{buffer} \mathrm{at} \mathrm{room} \mathrm{temperature.} \mathrm{The}$

242 injector was washed twice between injections. The column method run time was 10 minutes at room temperature, under isocratic conditions where the mobile phase was $1 \mathrm{x}$ PBS pH 7.4

244 at a flow rate of $1 \mathrm{ml} \mathrm{min}{ }^{-1}$. Eluted protein was monitored at multiple wavelengths using a Multiwavelength detector (Jasco MD-2010 Plus). The column was calibrated using a set of

246 protein standards covering a range of known molecular weights (Blue Dextran (2000

$247 \mathrm{kDa})$,Thyroglobulin (Bovine) (669 kDa), IgG (Bovine) (150 kDa), Serum Albumin (Bovine) 66kDa, Ovalbumin (Chicken) 44 kDa, Lysozyme (Chicken) $14.5 \mathrm{kDa}$. 
For Food Hydrocolloids: Investigation of bovine serum albumin denaturation

249 The procedure followed was a modification of the methods of Whitaker (1963) and Andrews

250 (1964). Void volume $\left(V_{\mathrm{o}}\right)$ was determined by recording the elution volume (retention time)

251 of Blue Dextran at $280 \mathrm{~nm}$. The elution volume $\left(V_{\mathrm{e}}\right)$ of each protein in the $M_{\mathrm{w}}$ marker mixture

252 was determined at $280 \mathrm{~nm}$ by observing the retention time recorded by the instrument

253 software.

254 A standard curve was created by plotting the $\log _{10}$ of the $M_{\mathrm{w}} \mathrm{vs} . V_{\mathrm{e}} / V_{\mathrm{o}}$ for each protein in the 255 mixture. $V_{\mathrm{e}} / V_{\mathrm{o}}$ values were calculated for the unknown samples and the standard curve used 256 to determine the estimated $M_{\mathrm{w}}$.

2573 Results

$258 \quad 3.1$ Determination of Acoustic Parameters

259 As indicated by Equation 3 above, it is necessary to measure the dependence of the velocity

260 of longitudinal sound on the volume fraction of the protein in solution, in order to determine

261 the molecular compressibility. Studies of concentration dependence were carried out on BSA

262 solutions and density measurements as previously reported in Apenten et al (2000) were used.

263 The partial molar volume, effective molecular density, and effective molecular radius for

264 BSA were obtained from these data using a molecular weight of 66500 Da (Murayama \&

265 Tomida, 2004) and are given in Table 2. These data were then used to calculate volume

266 fraction.

267 Velocity measurements taken in the ICHOS II, over the range $20-160 \mathrm{MHz}$ and a

268 concentration range up to $40 \mathrm{mg} \mathrm{ml}^{-1}$ showed no frequency dependence. The concentrations

269 were converted to volume fraction using the partial molar volume, and a single mean value of 
For Food Hydrocolloids: Investigation of bovine serum albumin denaturation

270 velocity taken for each concentration. Figure 2 shows the inverse of the velocity relative to 271 water plotted against volume fraction, following the method of Equation 5.

272 A linear relationship is clearly suitable for this experimental data. Hence, it is appropriate to neglect the multiple scattering contribution to velocity at these concentrations. The gradient of the straight-line fit is the parameter $C_{v}$ (Equation 6). If thermal contributions are neglected initially and we assume that molecular relaxation is insignificant, the velocity gradient $C_{v}$ relates only to the compressibility and density difference between water containing buffer and the BSA molecules in solution. Since the density of the BSA molecules has been determined from the solution density measurements (Table 2), the compressibility of the BSA molecules can be found (Equation 7): this is the molar adiabatic compressibility for BSA and lumps together the intrinsic compressibility of the protein chain, the compressibility of any cavities within the protein structure arising from its tertiary structure and solvent interactions with the protein which are also a function of solvent accessible surface (- see discussion below). Its

283 value of $\kappa^{\prime}=2.05 \times 10^{-10} \mathrm{~Pa}^{-1}$ (corresponding to a longitudinal sound velocity for the 284 hydrated BSA molecule of $1920 \mathrm{~ms}^{-1}$ ) and our previously obtained value for BSA of $1.2 \times 10^{-}$ ${ }^{10} \mathrm{~Pa}^{-1}$ compare with the value for water of $4.48 \times 10^{-10} \mathrm{~Pa}^{-1}$. Our previous work indicated that the compressibility was very sensitive to $\mathrm{pH}$ and to the thermal history of the protein; the experiments reported here were carried out isothermally, whereas our earlier experiments 288 involved scanning the temperature at $20{ }^{\circ} \mathrm{C} \mathrm{h}^{-1}$. Our value $\left(\kappa^{\prime}=2.05 \times 10^{-10} \mathrm{~Pa}^{-1}\right)$ for the 289 adiabatic compressibility of the hydrated molecule between $0 \mathrm{mg} \mathrm{ml}^{-1}$ and $40 \mathrm{mg} \mathrm{ml}^{-1}$ can be 290 compared with the value of $0.89 \times 10^{-10} \mathrm{~Pa}^{-1}$ given by Chalikian (1996) for the 'apparent 291 specific adiabatic compressibility' (The hydrated value, not the intrinsic value) for BSA at a 292 single concentration of $3 \mathrm{mg} \mathrm{ml}^{-1}$. The Chalikian (1996) method involved subtracting the 293 velocity of sound in the solvent from that in the $3 \mathrm{mg} \mathrm{ml}^{-1}$ solution, this is called the velocity Page 13 of 29, printed 14/11/201016:04:35 
For Food Hydrocolloids: Investigation of bovine serum albumin denaturation

294 increment. On the other hand, our method plots the inverse square velocity against

295 concentration over the entire concentration range (Figure 2), a much more accurate 296 procedure. The scattering theory calculation estimates the contribution of thermal scattering

297 to the compressibility of $4 \%$, much less than the estimate made in our earlier work by the

298 frequency independent, temperature scanning method. The compressibility value determined

299 by this method is the property of the scatterer of sound, which is the BSA molecule and any

300 modified water surrounding it (hydration layer). Scattering theory does not independently

301 provide a method for identifying the contribution of hydration and intrinsic compressibility

302 terms, but does identify the likely effect of thermal scattering in the compressibility

303 calculation. Given the nature of the temperature dependence of the compressibility (Figure 3

304 in Apenten et al. (2000)), the thermal scattering contribution may have a big relative impact

305 on compressibility close to the melting temperatures $\left(T_{\mathrm{m} 1}\right.$ and $\left.T_{\mathrm{m} 2}\right)$ because here the

306 compressibility is small and the thermal contribution may therefore be a large proportion of

307 the total compressibility. Comparison of the two sets of the experiments indicates that there

308 may be a significant time dependent and hysteresis component in the compressibility. As

309 discussed below, we can also expect the entropic contribution from the hydrophobic groups

310 coming in contact with water as the molecule unfolds to have an impact on compressibility.

311 Holmes and Challis' (1996) data indicate that at the relaxation peak of $20 \mathrm{MHz}$;

312 approximately half of the attenuation relative to water is due to relaxation processes at $37{ }^{\circ} \mathrm{C}$.

313 The attenuation data for the BSA solution at $25^{\circ} \mathrm{C}$ is shown in Figure 3. We have not

314 attempted to separate out the contributions of relaxation and scattering, due to the complexity

315 of the problem. The attenuation for the molecules is determined from Equation 4, through a

316 plot of attenuation against concentration of BSA, where we have combined together

317 relaxation contribution and scattering contributions. It is notable that the attenuation of the 
For Food Hydrocolloids: Investigation of bovine serum albumin denaturation

318 molecules is very large compared with water. At $160 \mathrm{MHz}$, the attenuation in water is

$319 \sim 560 \mathrm{Npm}^{-1}$, but for the BSA molecules, it is $\sim 5200 \mathrm{Npm}^{-1}$, almost a factor of 10 higher. The

320 excess attenuation of the solution over water was nearly $90 \mathrm{Npm}^{-1}$ at the highest

321 concentration, which corresponded to a volume fraction of 0.03 (or $3 \% \mathrm{v} / \mathrm{v}$ ).

322

\subsection{Oligomerisation (Protein-protein interaction)}

323 The BSA samples were heated in such a way that all stages of the 324 oligomerisation/aggregation process of the protein could be studied. The ICHOS II 325 instrument had the advantage that it requires only a few millilitres of sample. Figure 4 326 contains data from three replicated experiments and shows that aggregation can be 327 reproducibly induced in $\mathrm{BSA}$ at $60{ }^{\circ} \mathrm{C}$ increasing the particle size from approximately $8 \mathrm{~nm}$ 328 to approximately $21 \mathrm{~nm}$ within one hour, according to DLS measurements (insert in Figure 329 4). Further treatment up to $3 \mathrm{~h}$ appears to have no further effect on particle size, although the 330 higher resolution SEC data measured at $70{ }^{\circ} \mathrm{C}$ (Figure 5, Figure 6) indicates that the amount 331 of monomer and dimer continue to decrease with an increase of the trimer; nevertheless, only

332 the monomer, dimer and trimer are present in significant quantities. It is unlikely that 333 significant denaturation is occurring in these experiments because no change in the velocity 334 of sound was seen, consistent with studies in HSA (Mitra, Sinha, \& Pal, 2007) and an 335 unchanging molecular conformation.

\section{$336 \quad 3.3 \quad$ Heat denaturation of BSA}

337 This part of the study presented a challenge due to the large quantities of protein (500 $\mathrm{ml})$

338 required by the Malvern Ultrasizer. The Ultrasizer was nevertheless included because it 339 gives absolute attenuation values, useable as a reference. Thus, it was necessary to heat large 340 quantities and this was achieved using canning techniques for which a typical heating curve is Page 15 of 29, printed 14/11/201016:04:35 
For Food Hydrocolloids: Investigation of bovine serum albumin denaturation

341 shown in Figure 1. The resultant attenuation spectra and DLS data are shown in Figure 7,

342 Figure 8, together with an estimate of the relative proportions of monomer, dimer and trimer

343 in the $20 \mathrm{~nm}$ peak (Table 4). It is clear that aggregation of the BSA is manifested in these

344 data, showing an increase in attenuation with heating. Little change in the main peak in the

345 DLS data is seen, in comparison with the control sample for the first three minutes. After

346 four minutes the size of the main peak begins to increase and becomes more scattered with a

347 distinct increasing trend in size. The secondary peak appears after ten minutes then rapidly

348 rises for the rest of the experiment. Associated changes in the velocity of sound and density

349 (not shown) are too small to explain the changes in the attenuation spectra. However,

350 significant changes in the velocity of sound occurred at the point where it became impossible

351 to carry out attenuation spectroscopy and DLS measurements, probably due to the

352 introduction of a frame modulus to the elastic modulus, in addition to compressibility, arising

353 from gelation (see later discussion). On heating at $80{ }^{\circ} \mathrm{C}$ for $100 \mathrm{~s}$, the initial BSA solution

354 becomes yellow and translucent, then more cloudy. After $1000 \mathrm{~s}$ at $80{ }^{\circ} \mathrm{C}$, the sample

355 becomes gel like, and becomes stiffer with heating time.

356 The storage modulus $G^{\prime}$ increases with heating time and approaches $G^{\prime \prime}$ at roughly two

357 minutes (Figure 9). The DLS data (Figure 8) indicates the appearance of large aggregates

358 after 10 minutes. Between 100 and 300 seconds, $G^{\prime}$ increased rapidly signifying that

359 aggregates were forming in solution, then after ten minutes gelation occurred, coinciding with

360 the rapid rise in the secondary DLS peak in Figure 8. Then $G^{\prime}$ kept on increasing with the

361 time of cooking, indicating that gelation continued to develop (Baier, Decker, \&

362 McClements, 2004). The fact that $G^{\prime}$ continues to increase throughout 2 hours shows that the

363 gel formation was not completed and that the structure of the gel was still evolving. Neither

364 DLS nor attenuation spectroscopy could be performed after roughly ten minutes at $80{ }^{\circ} \mathrm{C}$, in

Page 16 of 29, printed 14/11/201016:04:35 
For Food Hydrocolloids: Investigation of bovine serum albumin denaturation

365 the case of the Ultrasizer; this was because the transducer movement broke up the gel

366 structure. Whilst this problem does not present in the case of the ICHOS II instrument,

367 measurements were only carried out on samples that did not gel. No measurements were

368 possible on the BSA sample heated at $105{ }^{\circ} \mathrm{C}$ because it was not possible to insert the solid

369 sample into the ultrasound measurement cells, which are designed to take liquids.

370 The DLS data (Figure 8) indicates that dimerisation and trimerisation (Table 4) dominates the

371 first 4 minutes of 'cooking', followed by the rapid increase in large aggregates. However, it

372 was not possible to resolve dimers from trimers based on the DLS data. Attenuation shows 373 an increasing trend with cooking time, presumably as it responds (Figure 10) to the 374 aggregation process.

\section{Discussion}

\section{$376 \quad 4.1$ Relaxation}

377 BSA has been the subject of significant attention in ultrasound studies (Kessler \& Dunn, 378 1969; Zana \& Lang, 1970; Hussey \& Edmonds, 1971; Lang, Tondre, \& Zana, 1971;

379 Kremkau \& Cowgill, 1985; Barnes, Evans \& Lewis, 1985; Kremkau, 1988; Bryant \& 380 McClements, 1999), presumably because the quantities required to make accurate attenuation 381 measurements necessitated a readily available protein. At lower frequencies than our range 382 (Barnes et al. (1985, 1986, 1988), some absorption peaks indicate conformational changes 383 and proton transfer at amino groups.

384 In the frequency range of the experiments reported in this work, most absorption was 385 previously attributed to proton transfer equilibria (Kessler and Dunn (1969), Dunn \& Kessler, 386 (1970), Barnes et al.(1985) Choi, Bae \& Takagi (1990), Holmes and Challis (1996)) These 
For Food Hydrocolloids: Investigation of bovine serum albumin denaturation

387 workers carried out a variety of relaxation frequency fitting. In contrast, in our work no

388 absorption peaks were observed. We analyse the attenuation spectra as a scattering 389 phenomenon, where relaxation effects are lumped together in the apparent attenuation 390 properties of the solute (i.e. in the frequency squared dependence of the attenuation 391 coefficient).

392 Mitra, Sinha \& Pal (2007), recently reported measurements in the temperature range $20{ }^{\circ} \mathrm{C}$ to $39370{ }^{\circ} \mathrm{C}$, on human serum albumin (HSA) using a range of techniques including ultrasound, 394 which suggest a rapid loss in $\alpha$-helicity beyond $55^{\circ} \mathrm{C}$ but no observable change in secondary 395 and tertiary structure below this temperature.

396 It is apparent from these studies, that relaxation processes make a significant contribution to 397 the molecular attenuation of sound, in addition to scattering effects. The separation of the 398 contributions of relaxation and scattering is non-trivial and is the subject of current studies in 399 our laboratory. Whilst, in the case of ultrasound attenuation, we have not separated out the 400 relaxation contribution and have studied the ultrasonic data in the context of scattering by the 401 molecules, we have shown above that in the case of BSA, the dependence of the velocity of 402 sound is accurately explained by a scattering model (Figure 2).

\section{$403 \quad 4.1$ Denaturation}

404 When the BSA solution is heated, the protein molecules unfold, the compact structural form 405 of the molecule is disrupted. The globular proteins undergo conformational changes that 406 allow hydrophobic groups or groups containing sulfhydryls, to be exposed. Protein 407 aggregation and gel formation occur when the protein surface activity is sufficient. According 408 to Clark, Kavanagh \& Ross-Murphy (2001), heat-induced gelation of BSA is considered a 409 multistage process that involves three main stages. The first step is the initial protein 
For Food Hydrocolloids: Investigation of bovine serum albumin denaturation

410 unfolding under heating which leads to a more reactive structural form. Sometimes

411 dimerisation happens. The second step is the formation of linear fibril aggregates via

412 disulfide and hydrophobic bonding. The aggregates form by nucleation and growth. The third

413 step is a random cross-linking of the fibrils via non-covalent interaction. The association of

414 the fibrils follow a random poly-condensation process that generates many species. The BSA

415 gels show a strong inter-fibril connection. The visual observation of the samples after heating

416 gives an idea of the nucleation and growth of the aggregates, because the more the samples

417 were heated, the more it was possible to see bigger particles. The random poly-condensation

418 of the fibrils can also explain a big variation in the size distribution of particles even if

419 globally the size of the BSA particles increases with heating.

420 Boye, Kalab, Alli, \& C.Y. (2000) on the other hand showed that a BSA gel is made of a 421 network of small BSA aggregates of different shapes and sizes in the nanometre scale. These 422 aggregates form through both disulfide and hydrophobic bonding. When native BSA 423 molecules are dispersed in phosphate buffer at neutral $\mathrm{pH}$, they do not form gels because the attractive interactions, which are principally van der Waals, hydrogen bonding, and hydrophobic interactions, are weaker than the repulsive interactions, which are mostly electrostatic, hydration and configurational entropy (Baier, Decker \& McClements, 2004).

427 This point of view is more in accord with our results than the linear fibril view of Clark, 428 Kavanagh and Ross-Murphy, although we cannot exclude the formation of linear fibrils in the 429 final stages of gel formation.

430 The heat- or pressure-induced unfolding (denaturation) process is commonly treated as a two431 state model or transition comprising a native $(\mathrm{N})$ and unfolded $(\mathrm{U})$ state $\mathrm{N} \leftrightarrow \mathrm{U}$. This model 432 particularly applies to the unfolding of small monomeric globular proteins (Privalov, 1979), 433 for which intermediate states are neglected since they are considered so sparsely populated. Page 19 of 29, printed 14/11/201016:04:35 
For Food Hydrocolloids: Investigation of bovine serum albumin denaturation

434 The main driving forces for folding are the entropic contributions of free water and changes 435 in entropy due to release/binding of water molecules, affecting the hydrophobic core of 436 globular proteins. At ambient and physiological temperatures, unfolding carries a significant 437 entropic cost, particularly due to the presentation of hydrophobic side chains to the solvent.

438 In order to understand how denaturation of the BSA molecule may impact its acoustic 439 properties it is necessary to locate them in the preceding model of oligomerisation and 440 denaturation.

441 Sound velocity is determined for the solution by the molar compressibility, which includes 442 contributions from the intrinsic compressibility of the molecule and the modified 443 compressibility of the water surrounding the molecule (commonly known as the hydration 444 layer). Thermodynamically, the compressibility is related to free energy since isothermal 445 compressibility is the pressure gradient of the chemical potential (Chalikian, 2008). Whilst 446 the intrinsic compressibility is affected primarily by secondary structure, the hydration 447 contribution is determined largely by the solvent-accessible area of the molecule. Since we 448 measure adiabatic compressibility in acoustic experiments, an additional thermal term will be 449 present.

450 A number of workers have reported studies of protein denaturation processes using 451 ultrasound velocity and absorption techniques (Kremkau, 1987; Wang, Tolkach \& Kulozik, 452 2006). A detailed discussion of the relationship between solvent accessible area, intrinsic compressibility, and hydration compressibility is to be found in Apenten et al (2000) and 454 Chalikian (2008). Molecular compressibility is likely to change in step with structural 455 changes associated with denaturation of the molecule, although the free energy difference 456 between folded and unfolded states of BSA is relatively small, since enthalpic and entropic 457 terms are approximately equal but opposite in sign, so folding in itself is unlikely to Page 20 of 29, printed 14/11/201016:04:35 
For Food Hydrocolloids: Investigation of bovine serum albumin denaturation

contribute significantly to changes in compressibility. Our observation that the velocity of sound in the BSA molecule hardly changes during the initial stages of denaturation is 460 consistent with small changes in free energy, solvent accessible area and secondary structure.

461 On the other hand, the attenuation of the molecule is not so directly related to free energy and solvent accessible area. Rather it is controlled by scattering and relaxation, which appears much more sensitive to denaturation. The ultrasound scattering model developed from our 464 data indicates that particle size changes associated with oligomerisation (dimers, trimers, etc) are well below the size range where changes will impact on the ultrasound attenuation. We expect therefore, but cannot prove since we were unable to separate the effects of scattering and relaxation, that the attenuation changes we observe during oligomerisation arise due to relaxation effects,

Time- and frequency-independence of the sound velocity and density indicates that the compressibility of the weakly heated treated molecules is constant, consistent with an absence of significant changes to the primary, secondary and tertiary structure of the molecule.

473 Significant changes in the velocity of sound (not shown) were observed once gelation occurred, together with significant changes in elastic modulus (Figure 9). This may be expected since gelation arising from denaturation will add an additional term to the material compressibility due to the introduction of shear $(G)$ and frame (an additional compressional

477 modulus arising from molecule-molecule interactions in the solid state) moduli to elastic 478 modulus governing the propagation of compressional ultrasound waves. Referring to 479 Equations 1 and 2, Figure 9 and the determined value for the bulk modulus of water of $2.23 \mathrm{x}$ $48010^{9} \mathrm{~Pa}$, the shear modulus in the most denatured samples does not exceed $100 \mathrm{~Pa}$, far too 481 small a value to be measureable through the longitudinal wave velocity. This leaves Page 21 of 29, printed 14/11/201016:04:35 
For Food Hydrocolloids: Investigation of bovine serum albumin denaturation

482 contributions from the frame modulus and/or changes in molecular structure as possible

483 explanations for the changing velocity of sound under conditions of severe denaturation.

\section{Conclusions}

485 In the experiments described above, conditions were adjusted so that the initial stages of 486 dimerisation and trimerisation could be studied. Less severe heat treatment caused the

487 molecule to form dimers and trimers, detected by the ICHOS II system through changes in 488 attenuation spectra consistent with DLS and SEC observed particle size increase. In this 489 case, no velocity changes were observed; this is consistent with little or no molecular 490 conformation change.

491 BSA was then 'cooked', the protein underwent all the stages of oligomerisation, aggregation, 492 denaturation and gelation described above. Irreversible denaturation and gelation of the 493 protein affected both ultrasound attenuation spectra and the velocity of sound, consistent with 494 significant molecular conformation change and/or molecule-molecule interaction.

495 Ultrasound measurements are sensitive to all stages in the aggregation and denaturation of 496 BSA, responding to changes in the tertiary and quaternary structure. Aggregation led to 497 measurable changes in ultrasound spectra correlating with DLS sizes. During 498 oligomerisation, the BSA molecules continue to behave acoustically as individual particles, 499 exhibiting little change in their compressibility. It is only when significant denaturation 500 occurs that molecular compressibility may change significantly. The burst-rf, pulsed-wave 501 ICHOS II system was much more sensitive to protein aggregation than the quasi-continuous502 wave Ultrasizer system for reasons which are still unclear.

503 In this study we set out to elucidate a theory which allowed quantitative interpretation of 504 ultrasound data in terms of particle properties such as size and compressibility. This proved Page 22 of 29, printed 14/11/201016:04:35 
For Food Hydrocolloids: Investigation of bovine serum albumin denaturation

505 difficult because (a) proteins both scatter sound and exhibit relaxation and (b) the interaction

506 between the protein and water is a complicated one involving solvent accessible area, cavities

507 and entropic hydrophobic interactions during folding, unfolding and oligomerisation. A

508 significant question is why ultrasound attenuation detects dimers and trimers in a region

509 where scattering predicts that it is insensitive to particle size? This is likely to be because

510 oligomerisation changes molecular relaxation, without altering the acoustic scattering cross-

511 section significantly.

512 In summary, concentration dependent ultrasound velocity spectroscopy, together with

513 densitometry uniquely measures a key parameter which is characteristic of protein structure,

514 the molecular adiabatic compressibility. This can be related to solvent accessible surface area,

515 hence providing insight into protein-solvent interactions. A novel aspect of our study is the

516 demonstration that ultrasound attenuation spectroscopy is sensitive to protein-protein

517 interactions such as dimerisation, trimerisation and stages of denaturation including gelation.

518 Together, velocity and attenuation spectroscopy reveal details of protein-protein and protein-

519 solvent interactions at the relatively high protein concentrations found in foods. This can give

520 a unique insight into the relationship between protein structure and function in foods and

521 many other protein containing systems, including biological systems. Future work in this area

522 requires the separate identification of relaxation and scattering contributions to acoustic

523 propagation and comparison between the measured molecular adiabatic compressibility and

524 that determined from molecular modelling. 
For Food Hydrocolloids: Investigation of bovine serum albumin denaturation

526 Acknowledgements

527 The project was funded by the UK DIUS (formerly DTI) as part of the National Measurement

528 System (NMS) Measurement for Biotechnology (MfB) 2004 - 2007 programme. Thanks are

529 due to Dr Caroline Orfila for carefully reviewing the paper and providing helpful suggestions.

\section{References}

531 Andrews, P., (1964) Estimation of the molecular weights of proteins by Sephadex gel532 filtration.Biochemical Journal, 91(2), 222-233.

533 Apenten, R. K., Buttner, B., Mignot, B., Pascal, D., \& Povey, M. J. (2000). Determination of 534 the Adiabatic Compressibility of Proteins in Concentrated Solution by a New Ultrasonic 535 Method. Food Hydrocolloids , 14 (1), 83-91.

536 Atkins, P. W., \& de Paula, J. (2002). Atkins' Physical Chemistry, 7th ed. Oxford: Oxford 537 University Press.

538 Baier, S. K., Decker, E. A., \& McClements, D. J. (2004). Impact of glycerol on thermostability and heat-induced gelation of bovine serum albumin. Food Hydrocolloids , 18, $540 \quad 91-100$.

541 Barnes, C., Evans, J. A., \& Lewis, T. J. (1988). Low-frequency ultrasound absorption in 542 aqueous-solutions of hemoglobin, myoglobin, and bovine serum-albumin - the role of 543 structure and pH. Journal of the Acoustical Society of America, 83, 2393-2404.

544 Barnes, C., Evans, J. A., \& Lewis, T. J. (1985). Ultrasonic-absorption of bovine serum545 albumin solutions in the frequency-range $200 \mathrm{kHz}-1 \mathrm{MHz}$. Journal of the Acoustical Society 546 of America, 78, 6-11. 
For Food Hydrocolloids: Investigation of bovine serum albumin denaturation

547 Barnes, C., Evans, J. A., \& Lewis, T. J. (1986). Ultrasonic-absorption of bovine serum548 albumin solutions in the frequency-range 60 to $160 \mathrm{kHz}$. Journal of the Acoustical Society of 549 America, 80, 1291-1296.

550 Bilaniuk, N., \& Wong, G. S. (1996). Speed of sound in pure water as a function of 551 temperature (vol 93, pg 1609, 1993). Journal of the Acoustical Society of America , 99 (5), 5523257.

553 Bilaniuk, N., \& Wong, G. S. (1993). Speed of sound in pure water as a function of 554 temperature. Journal of the Acoustical Society of America , 93, 1609-1612.

555 Boye, J., Kalab, M., Alli, I., \& Ma, C. Y. (2000). Microstructural properties of heat-set whey 556 protein gels: Effect of pH. Lebensmittel-Wissenschaft Und-Technologie-Food Science and 557 Technology, $33(3), 165-172$.

558 Bryant, C. M., \& McClements, D. J. (1999). Ultrasonic spectroscopy study of relaxation and 559 scattering in whey protein solutions. Journal of the Science of Food and Agriculture , 79, $560 \quad 1754-1760$.

561 Chalikian, T. V., Totrov, M., Abagyan, R., Breslauer, K. (1996). The Hydration of Globular 562 Proteins as Derived from Volume and Compressibility Measurements: Cross Correlating 563 Thermodynamic and Structural Data. Journal of Molecular Biology, 260, 588-603.

564 Chalikian, T. V. (2008). On the molecular origins of volumetric data. Journal of Physical 565 Chemistry B , 112, 911-917.

566 Challis, R. E., Povey, M. J., Mather, M. L., \& Holmes, A. K. (2005). Ultrasound techniques 567 for characterizing colloidal dispersions. Reports on Progress in Physics, 68 (7), 1541-1637. 
For Food Hydrocolloids: Investigation of bovine serum albumin denaturation

568 Choi, P.-K., Bae, J.-R., \& Takagi, K. (1990). Ultrasonic spectroscopy in bovine serum569 albumin solutions. Journal of the Acoustical Society of America , 87, 874-881.

570 Clark, A., Kavanagh, G., \& Ross-Murphy, S. (2001). Globular protein gelation - theory and 571 experiment. Food Hydrocolloids , 15 (4-6), 383-400.

572

Dunn, F., \& Kessler, L. W. (1970). Further remarks on ultrasonic properties of bovine serum 573 albumin solutions. Journal of Physical Chemistry , 74 (13), 2736.

574 El Kadi, N., Taulier, N., Le Huérou, J. Y., Gindre, M., Urbach, W., Nwigwe, I., et al. (2006). 575 Unfolding and refolding of bovine serum albumin at acid $\mathrm{pH}$ : ultrasound and structural 576 studies. Biophysical Journal , 91 (9), 3397-3404.

577 Holmes, A. K., \& Challis, R. E. (1996). Acoustic absorption due to proton transfer in 578 solutions of proteins, peptides and amino acids at neutral $\mathrm{pH}$. Journal of the Acoustical 579 Society of America, 100 (3), 1865-1877.

580 Hussey, M., \& Edmonds, P. D. (1971). Proton-transfer reactions - mechanism for absorption 581 of ultrasound in aqueous solutions of proteins. Journal of Physical Chemistry , 75 (26), $4012-$ 5824019.

583 Kessler, L., \& Dunn, F. (1969). Ultrasonic investigation of conformal changes of bovine 584 serum albumin in aqueous solution. Journal of Physical Chemistry, 73 (12), 4256-4263.

585 Kremkau, F. W. (1987). Absorption of ultrasound in denatured proteins. Journal of the 586 Acoustical Society of America, 81, S80-S81.

587 Kremkau, F. W. (1988). Biomolecular absorption of ultrasound. 3. Solvent interactions. $588 \quad$ Journal of the Acoustical Society of America , 83 (6), 2410-2415. 
For Food Hydrocolloids: Investigation of bovine serum albumin denaturation

589 Kremkau, F. W., \& Cowgill, R. W. (1985). Biomolecular absorption of ultrasound. 1. 590 Molecular-weight. Journal of the Acoustical Society of America, 577, 1217-1221.

591 Lang, J., Tondre, C., \& Zana, R. (1971). Effect of urea and other organic substances on 592 ultrasonic absorption of protein solutions. Journal of Physical Chemistry, 75 (3), 374-379.

593 Mitra, R. K., Sinha, S. S., \& Pal, S. K. (2007). Hydration in protein folding: Thermal 594 unfolding/refolding of human serum albumin. Langmuir , 23 (20), 10224-10229.

595 Murayama, K., \& Tomida, M. (2004). eat-induced secondary structure and conformation 596 change of bovine serum albumin investigated by Fourier transform infrared spectroscopy. 597 Biochemistry, 43 (36), 11526-11532.

598 Ooi, T. (1994). Thermodynamics of protein-folding - effects of hydration and electrostatic 599 interactions. Advances in Biophysics , 30, 105-154.

600 Ooi, T., \& Oobatake, M. (1991). prediction of the thermodynamics of protein unfolding - the 601 helix coil transition of poly(l-alanine). Proceedings of the National Academy of Sciences of 602 the United States of America, 88 (7), 2859-2863.

603 Povey, M. J. (1997). Ultrasonic techniques for fluids characterization. San Diego: Academic 604 Press.

605 Povey, M. J., \& McClements, D. J. (1988). Ultrasonics in Food Engineering: Part I. 606 Introduction and Experimental Methods. Journal of Food Engineering , 8, 217-245.

607 Povey, M. J., Awad, T. S., Huo, R., \& Ding, Y. (2007). Crystallization in Monodisperse 608 Emulsions with Particles in Size Range 20-200 nm. In E. D. Leser, Food Colloids, Self 609 Assembly and Material Science (pp. 399-411). London: RSC Publishing. 
For Food Hydrocolloids: Investigation of bovine serum albumin denaturation

610 Privalov, P. L. (1982). Stability of proteins - proteins which do not present a single 611 cooperative system. Advances in Protein Chemistry, 35, 1-104.

612 Privalov, P. L. (1989). Thermodynamic problems of protein-structure. Annual Review of 613 Biophysics and Biophysical Chemistry, 18, 47-69.

614 Privalov, P. L., \& Tsalkova, T. N. (1979). Micro-stabilities and macro-stabilities of globular 615 proteins. Nature, 280 (5724), 693-696.

616 Saluja, A., Badkar, A. V., Zeng, D. L., Nema, S., \& Kalonia, D. S. (2007). Ultrasonic storage 617 modulus as a novel parameter for analyzing protein-protein interactions in high protein 618 concentration solutions: Correlation with static and dynamic light scattering measurements. 619 Biophysical Journal (1), 234-244.

620 Samarasinghe, S. D., Campbell, D. M., A., J., \& Jonas, J. (1992). Biochemistry , 31, 77736217778.

622 Wang, Q., Tolkach, A., \& Kulozik, U. (2006). Quantitative assessment of thermal 623 denaturation of bovine alpha-lactalbumin via low-intensity ultrasound, HPLC, and DSC. 624 Journal of Agricultural and Food Chemistry, 54 (18), 6501-6506.

625 Whitaker, J.R., (1963) Determination of Molecular Weights of Proteins by Gel Filtration of 626 Sephadex. Analytical Chemistry, 35(12), 1950-1953.

627 Zana, R., \& Lang, J. (1970). Effect of pH on ultrasonic absorption of aqueous solutions of 628 proteins. Journal of Physical Chemistry, 74 (13), 2734-2736.

629 Zhang, C. T., \& Chou, K. C. (1995). An analysis of protein-folding type prediction by seed630 propagated sampling and jackknife test. Journal of Protein Chemistry, 14 (7), 583-593. 


\section{Tables}

632 Table 1 Calculation of partial molar volume for BSA.

633 Table 2 Partial molar volume, density and radius of BSA molecules in solution, derived from 634 the solution density measurements in this work and by Chalikian (1996), including the used 635 relationships between molecule radius, partial molar volume, molecular density and 636 molecular weight.

637 Table 3 Properties of water and BSA solute molecules used in calculations

638 Table 4 Monomer and oligomer data for BSA (Figure 8). Gravimetric data in the first row is 639 taken from Table 2 and is computed on the assumption that only monomers exist. These data 640 were obtained before heating. The DLS diameters are taken from the measured peaks in 641 Figure 8. In the final row, the composition of material in the $20 \mathrm{~nm}$ peak is then estimated 642 assuming it is composed of monomer, dimer and trimer.

\section{Figure Captions}

644 Figure 1 Representative temperature-time plot for the BSA denaturation studies. Solid line 645 retort temperature; dashed line - in-can temperature.

646 Figure 2 Inverse square velocity of sound plotted against partial molar volume fraction of 647 BSA.

648 Figure 3 Attenuation relative to water, of BSA solution plotted versus frequency measured in 649 the ICHOS instrument, of BSA solution (dotted line) heated at $60{ }^{\circ} \mathrm{C}$ for $1 \mathrm{~h}$ and control (solid 650 line) for $10 \mathrm{~g} / \mathrm{L}$ at $\mathrm{pH} 7$

651 Figure 4 Attenuation relative to water plotted against frequency for BSA in aggregated and 652 monomer forms. Inset is the DLS plot of volume \% versus size. The standard error of the 653 attenuation measurements is $\pm 0.2 \mathrm{~Np} \mathrm{~m}^{-1}$ and data are plotted for three replicated 654 experiments.

655 Figure 5 SEC retention time versus absorbance for BSA heated to $70{ }^{\circ} \mathrm{C}$ for 60 min (closed 656 circles), $120 \mathrm{~min}$ (plus signs) and $180 \mathrm{~min}$ (inverted triangles), together with the unheated 657 control sample (squares).

658 Figure $6 \%$ total peak area plotted against heating time for the data of Figure 5. Peak 1

659 represents the trimer species. Peak 2 represents the dimer species and peak 3 represents the 660 monomer species.

661 Figure 7 Total attenuation spectra for $40 \mathrm{~g} / \mathrm{l}, \mathrm{pH} 7 \mathrm{BSA}$ solutions for 'cooking times' of 0 min $662 \square, 2 \min \square, 4 \min \boldsymbol{\Delta}, 6 \min \mathrm{X}, 8 \mathrm{~min} \circ$ and $10 \mathrm{~min} \bullet$.

663 Figure 8 DLS particle size distributions for $0 \mathrm{~min}$ (solid line), $4 \mathrm{~min}$ (dashed line), $8 \mathrm{~min}$ 664 (dotted line) and 12 min (dotted-dashed line); for the system of Figure 7.

665 Figure 9 Plot of $\mathrm{G}^{\prime}$ and $G^{\prime \prime}$ for BSA heated at $80{ }^{\circ} \mathrm{C}$.

666 Figure 10 Total attenuation of BSA solution plotted versus cooking time at $80{ }^{\circ} \mathrm{C}$ for $40 \mathrm{~g} / \mathrm{l}$ at $667 \mathrm{pH} 7$ and $115 \mathrm{MHz}$. 


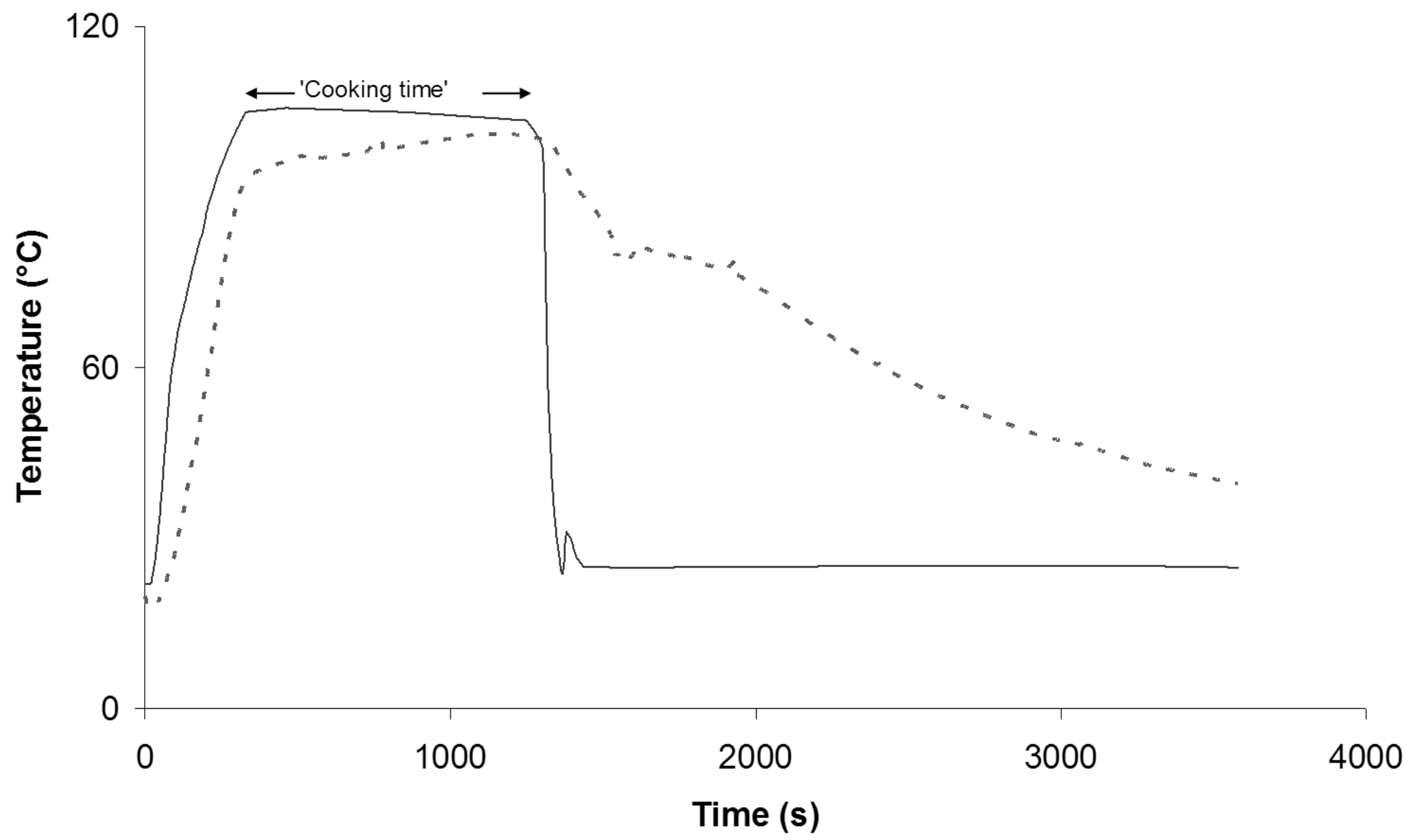




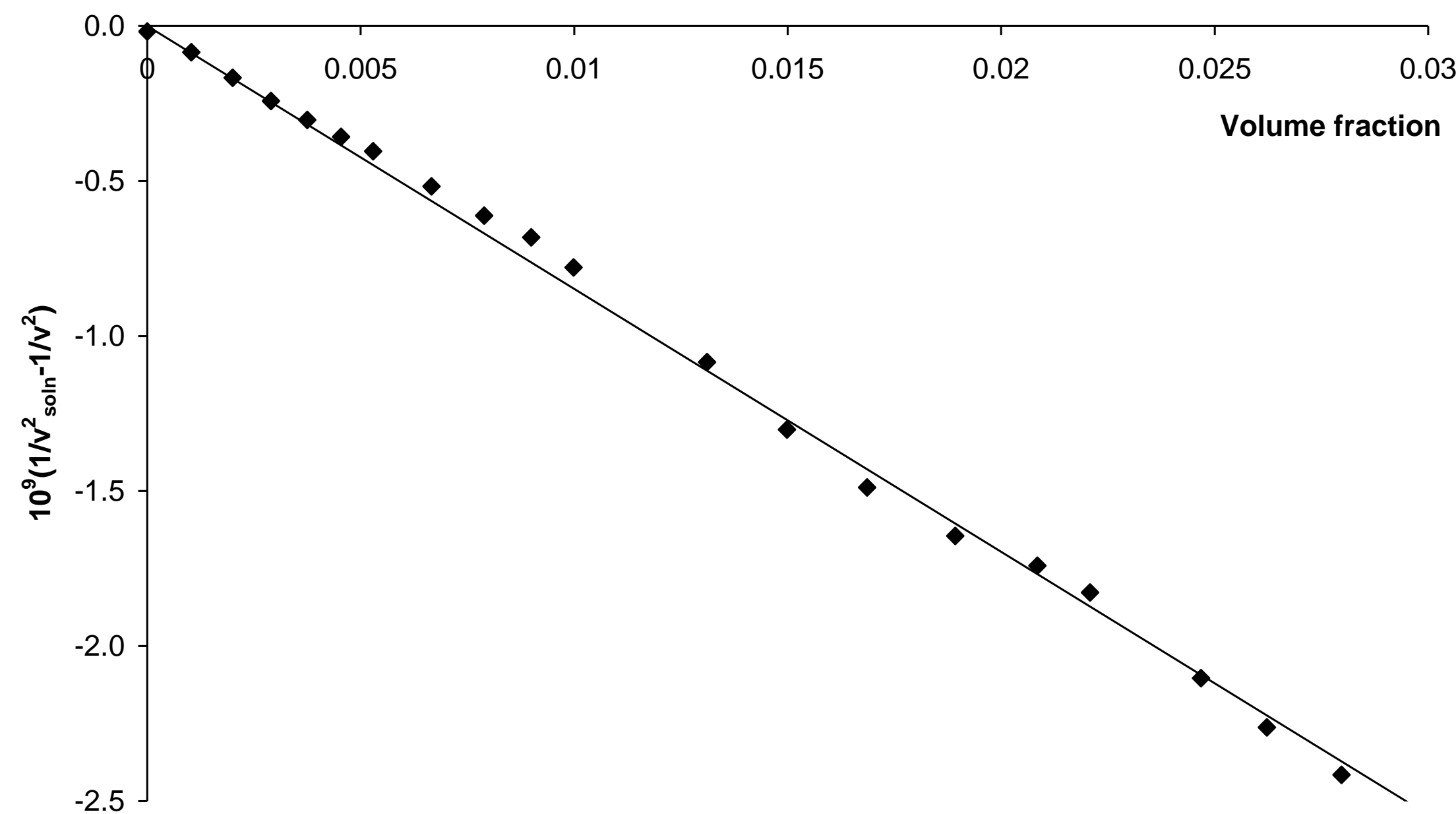




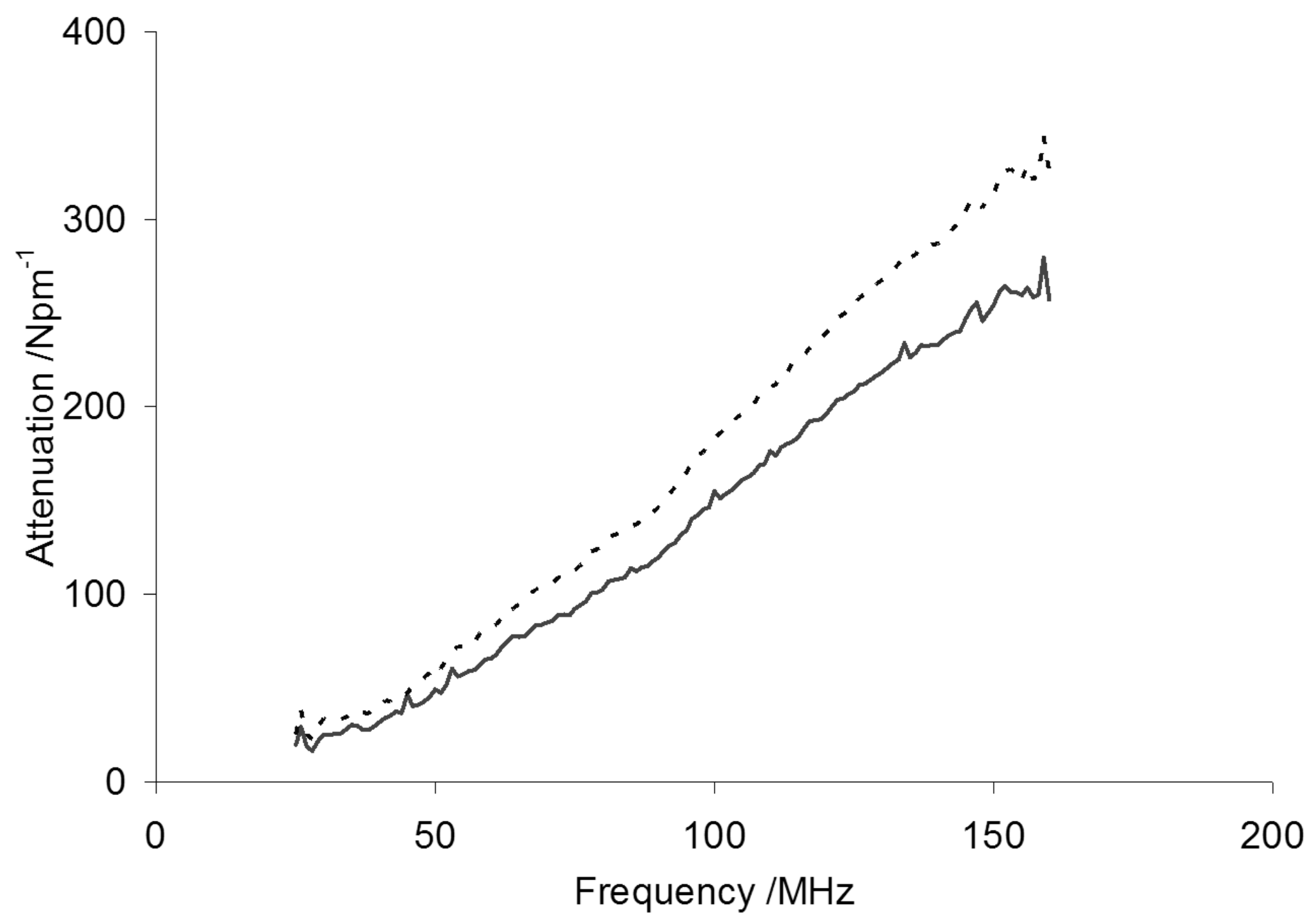


Relative attenuation $/ \mathrm{Np} \mathrm{m}^{-1}$

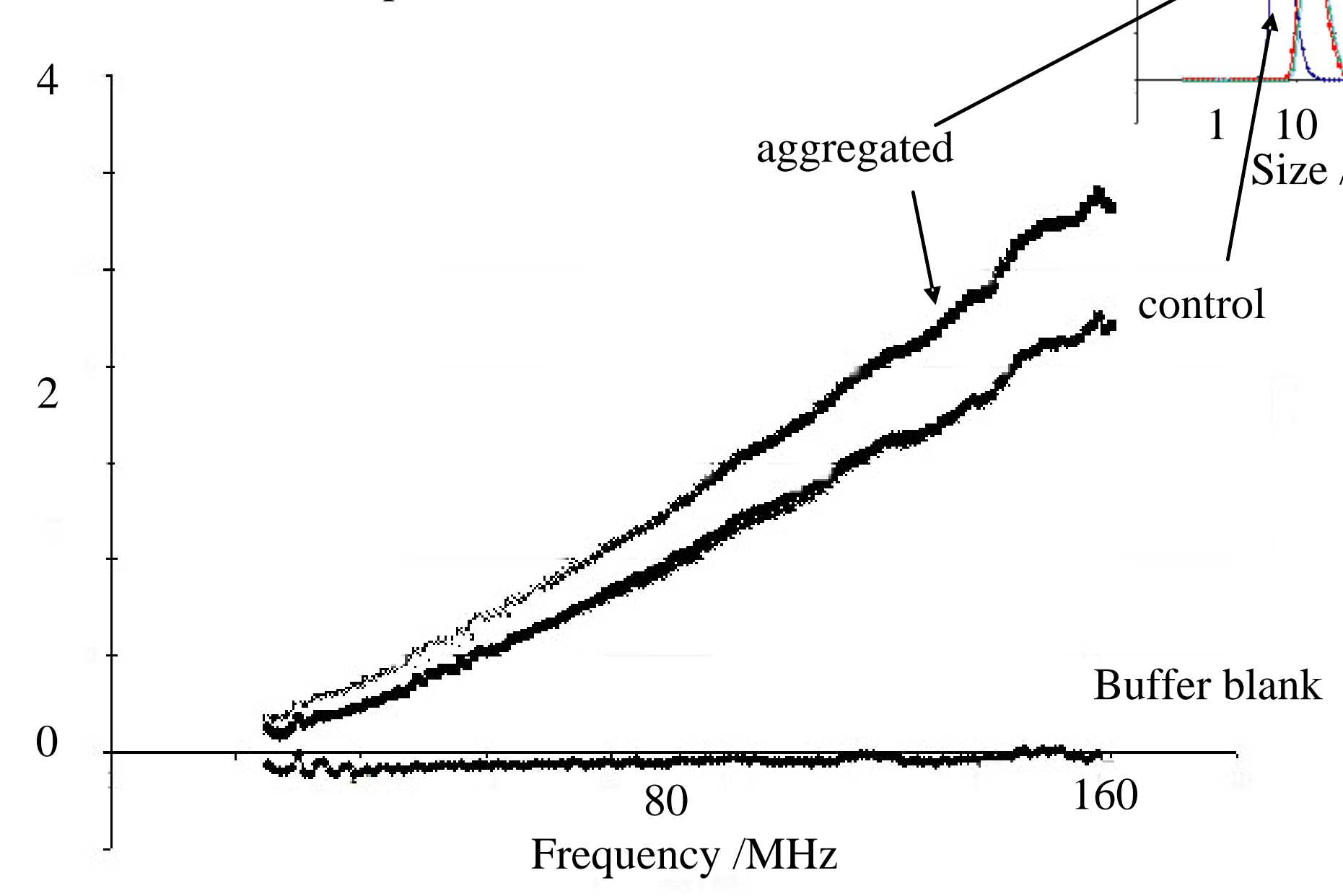




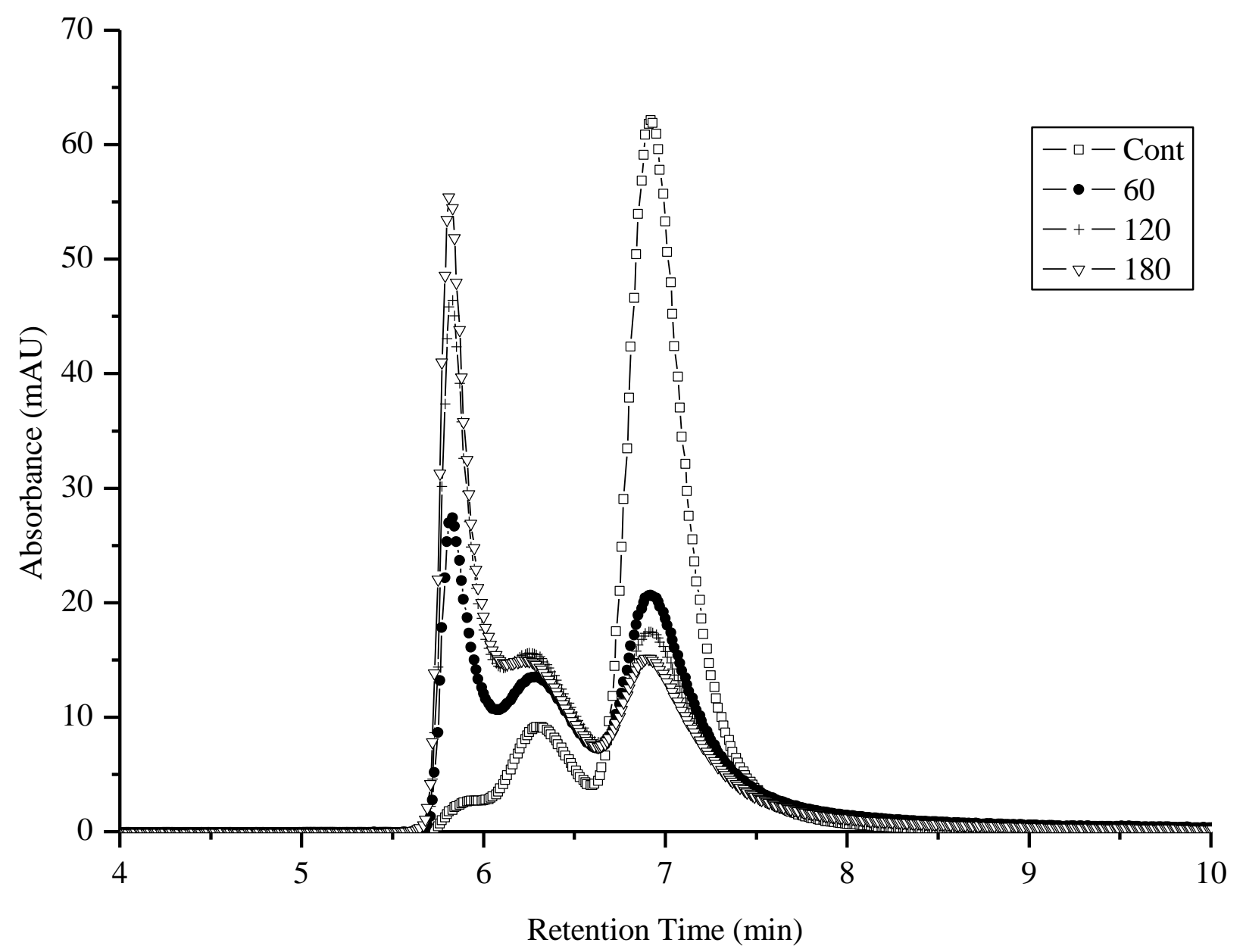




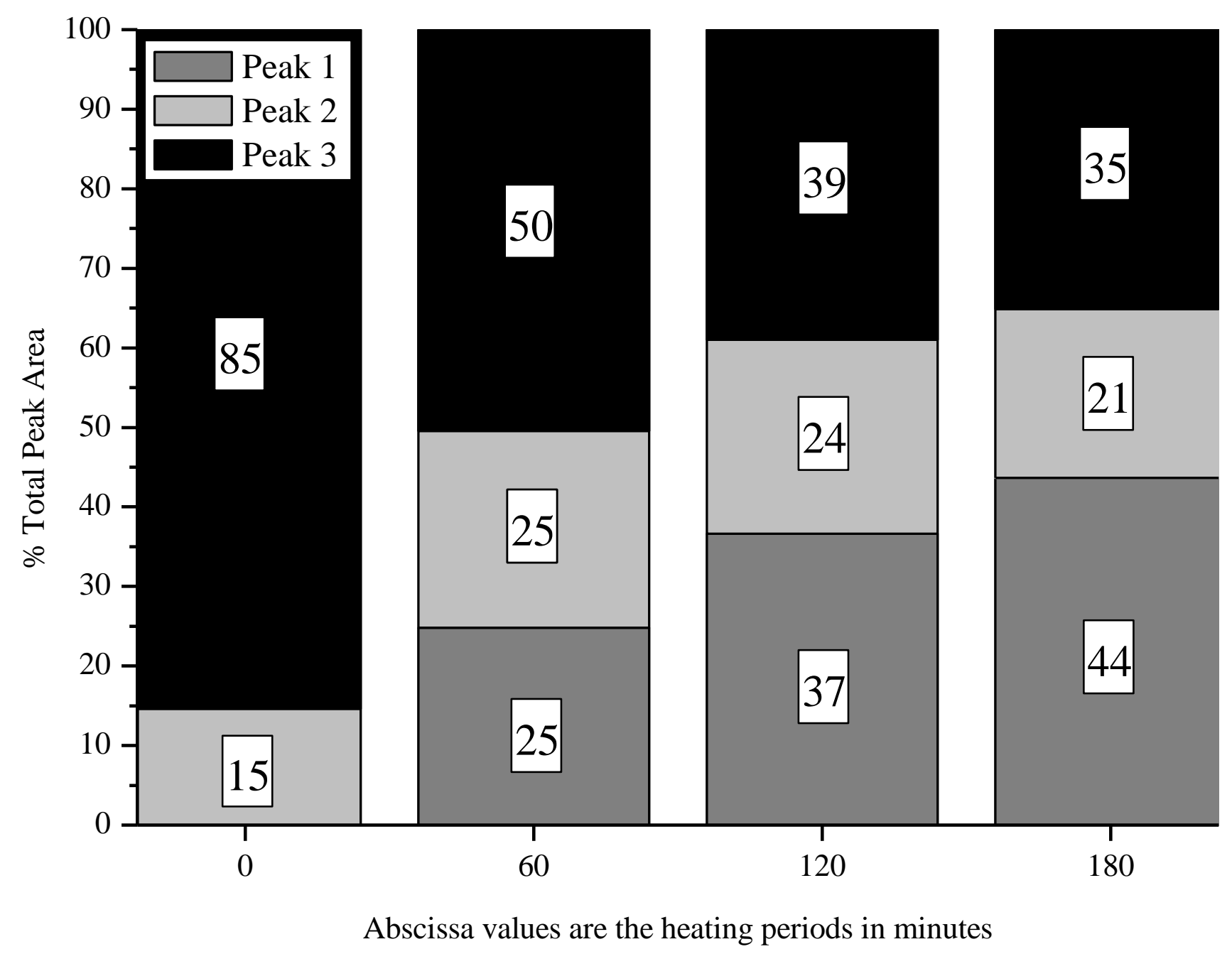




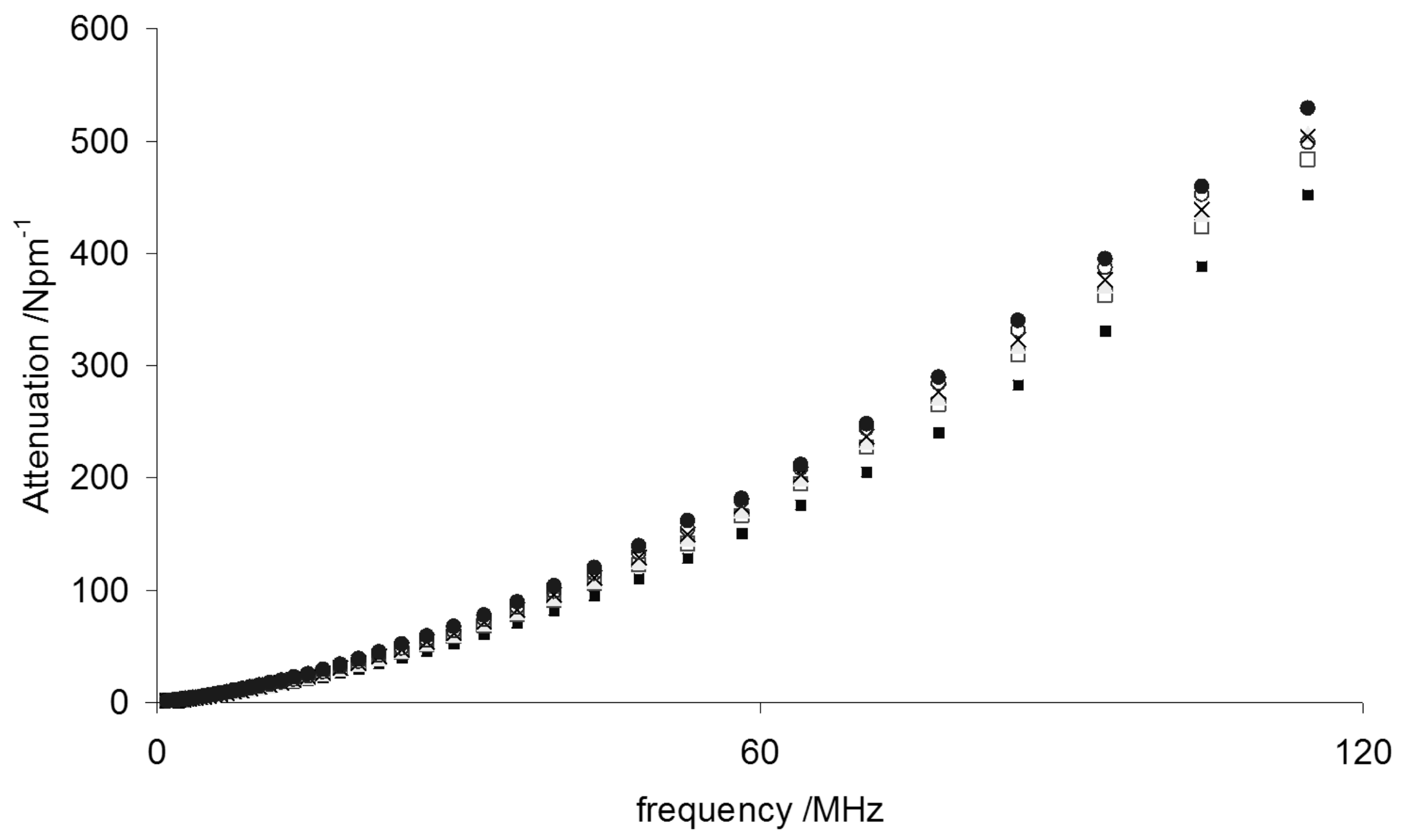




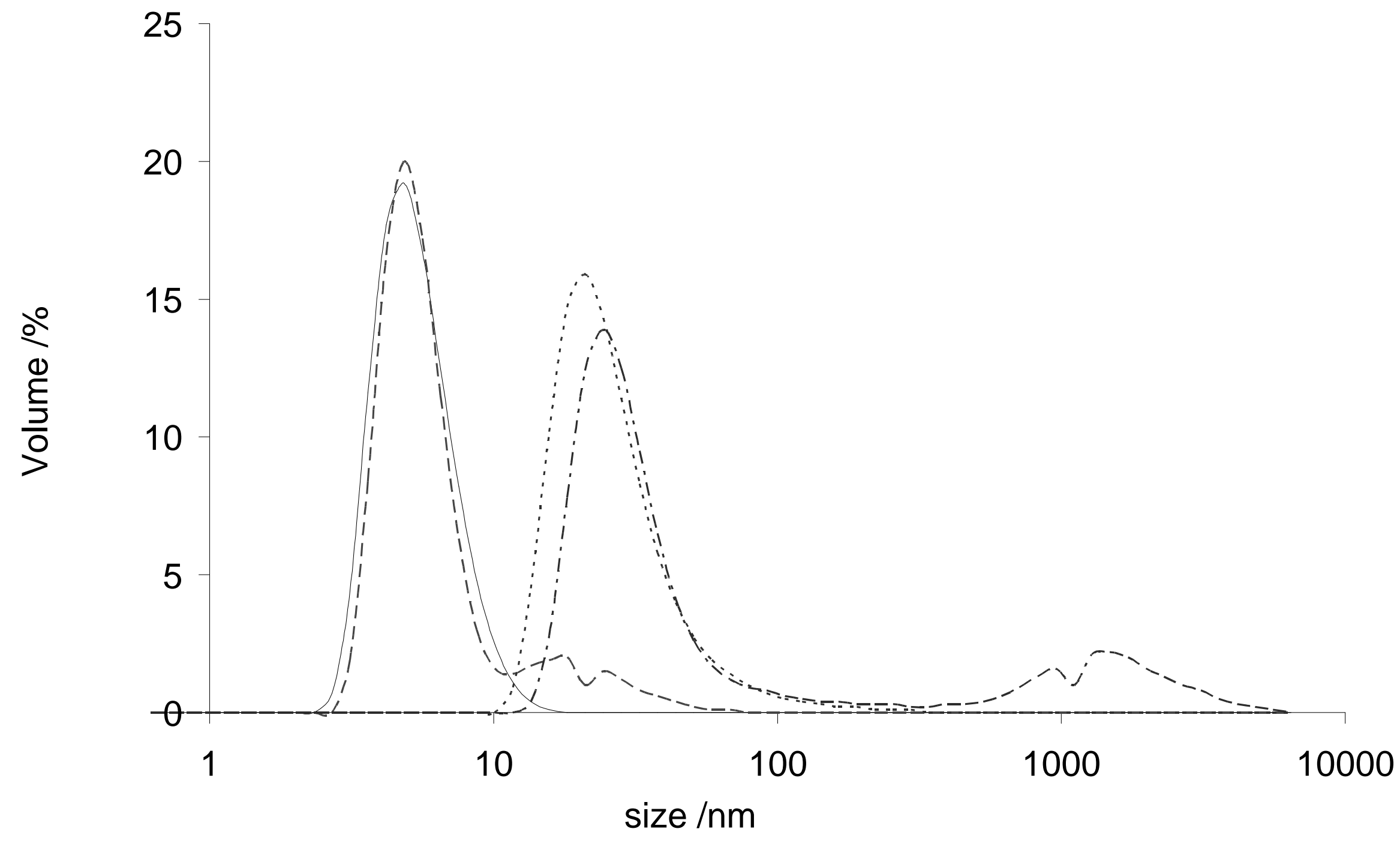




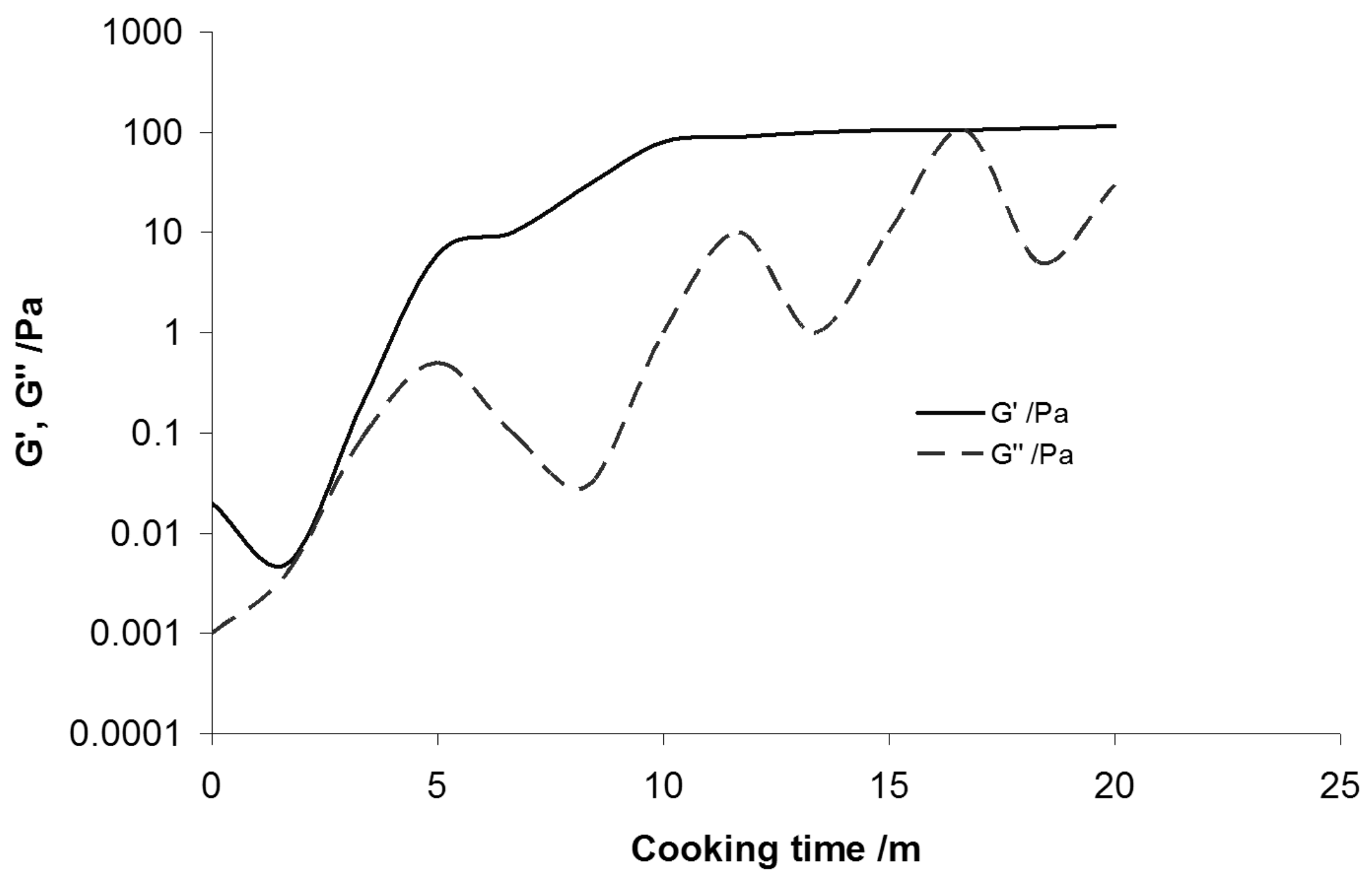




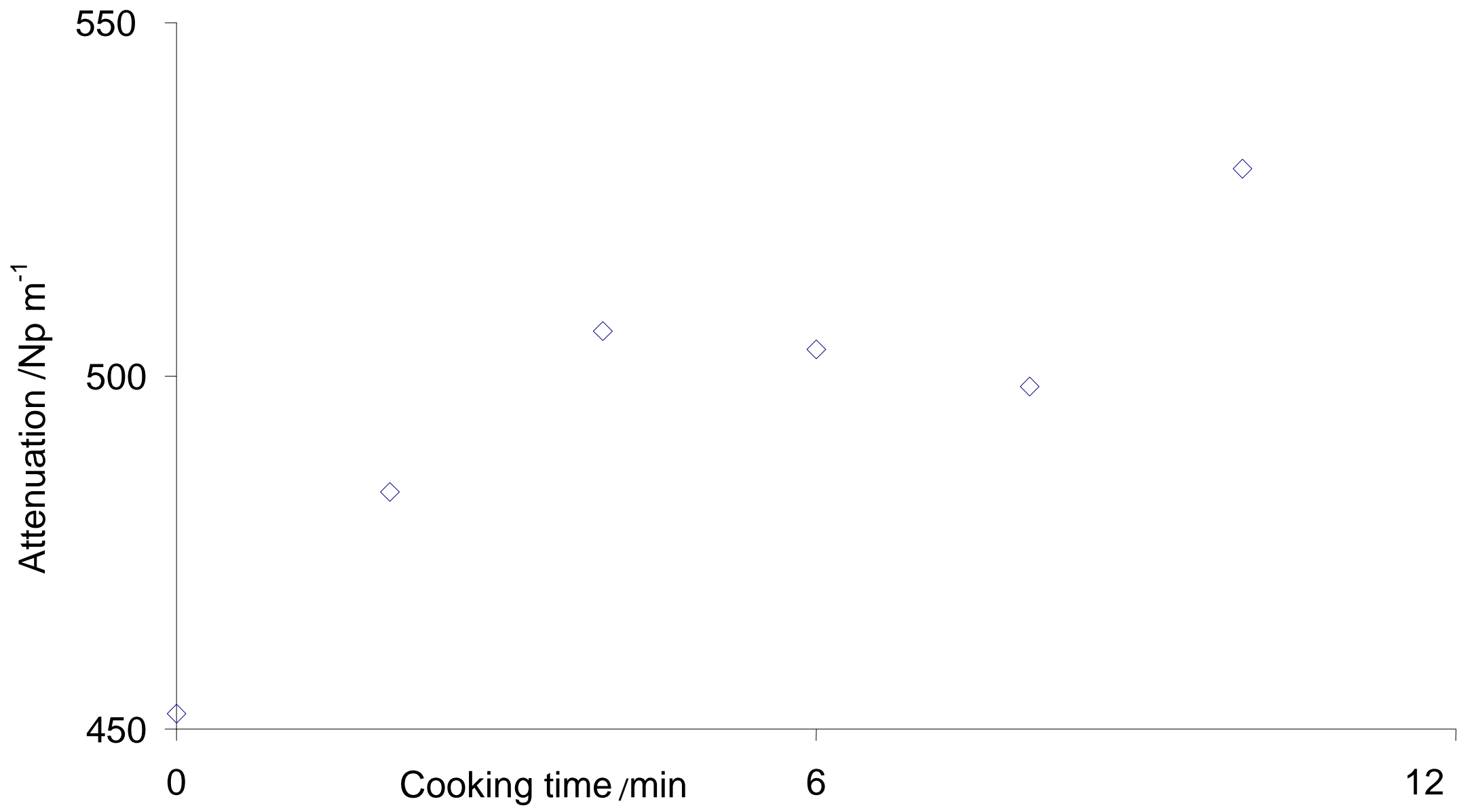




\section{Table 1}

\begin{tabular}{llll}
$\boldsymbol{c} / \mathbf{~} \mathbf{g ~ m L}^{-\mathbf{1}}$ & $\boldsymbol{\rho} / \mathbf{k g ~ m}^{-\mathbf{3}}$ & $\boldsymbol{V} / \mathbf{~} \mathbf{L}$ & $\boldsymbol{m}_{\mathbf{B S A}}$ \\
\hline 0 & 1002.8 & 997.2 & 0 \\
10 & 1005.1 & 1005.0 & 0.000151 \\
20 & 1007.9 & 1012.2 & 0.000304 \\
30 & 1010.5 & 1019.9 & 0.000460 \\
40 & 1012.6 & 1028.1 & 0.000618
\end{tabular}


Property

Value

This work

Chalikian (1996)

Partial molar volume $\tilde{V}_{\text {BSA }}$

$49823 \mathrm{~mL} \mathrm{~mole}^{-1}$

$49980 \mathrm{~mL} \mathrm{~mole}^{-1}$

Density $\rho_{\mathrm{BSA}}$

$1334.7 \mathrm{~kg} \mathrm{~m}^{-3}$

$1361 \mathrm{~kg} \mathrm{~m}^{-3}$

Radius of molecule $r$

$2.7 \mathrm{~nm}$

$2.7 \mathrm{~nm}$

Molecular weight MW

$66500 \mathrm{kDa}$

$68000 \mathrm{kDa}$

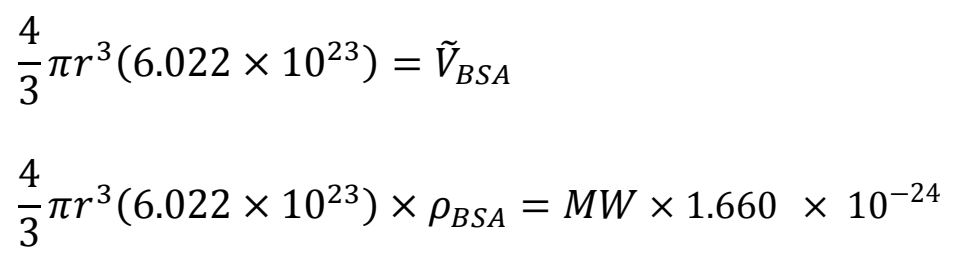




\begin{tabular}{lll} 
Property & Water & BSA solute \\
\hline Ultrasound velocity / $\mathrm{m} \mathrm{s}^{-1}$ & 1496.2 & 1334.7 \\
Density / $\mathrm{kg} \mathrm{m}^{-3}$ & 997.0 & 0.00042 \\
Thermal expansivity / $\mathrm{K}^{-1}$ & 0.00011 & \\
Viscosity / Pa s & 0.0009 & $1900^{\mathrm{a}}$ \\
Specific heat capacity / $\mathrm{J} \mathrm{kg}^{-1} \mathrm{~K}^{-1}$ & 4178 & $0.17^{\mathrm{a}}$ \\
Thermal conductivity / $\mathrm{W} \mathrm{m}^{-1} \mathrm{~K}^{-1}$ & 0.591 & \\
Attenuation exponent $p$ & 2 & \\
Attenuation factor / $\mathrm{Np} \mathrm{m} \mathrm{mHz}^{-1} \mathrm{MHz}^{-p}$ & 0.022 & \\
a Bryant \& McClements $(1999)$ & &
\end{tabular}




\begin{tabular}{rrrrrrr}
\multicolumn{2}{c}{ diameter } & volume & Volumi & Monomeric & dimeric & trimeric \\
Gravimetric & 5.4 & 82.44 & 3.25 & units & units \\
DLS monomeric & 8 & 268.08 & & 1 & \\
dimeric & 10.07 & 536.16 & 1 & \\
trimeric & 11.53 & 804.24 & 2 & & \\
DLS aggregate & 20 & 4188.79 & 3 & & \\
& & & & & & \\
\end{tabular}






\section{n \\ UNIVERSITY OF LEEDS}

14 November 2010

\section{Dear Editor,}

I am pleased to submit an extensively revised version of our paper entitled "Investigation of bovine serum albumin denaturation using ultrasonic spectroscopy" for consideration for publication in Food Hydrocolloids.

Best wishes,
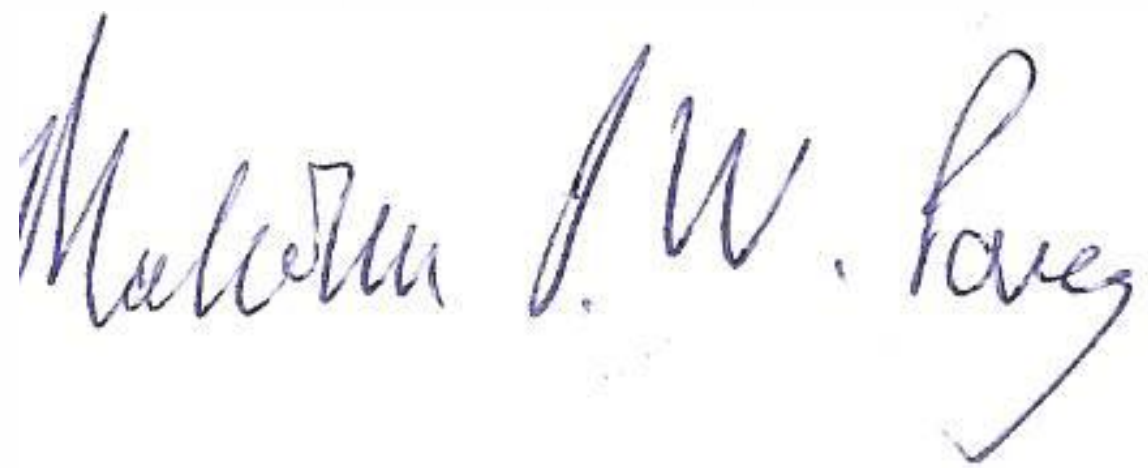

\section{Malcolm Povey}

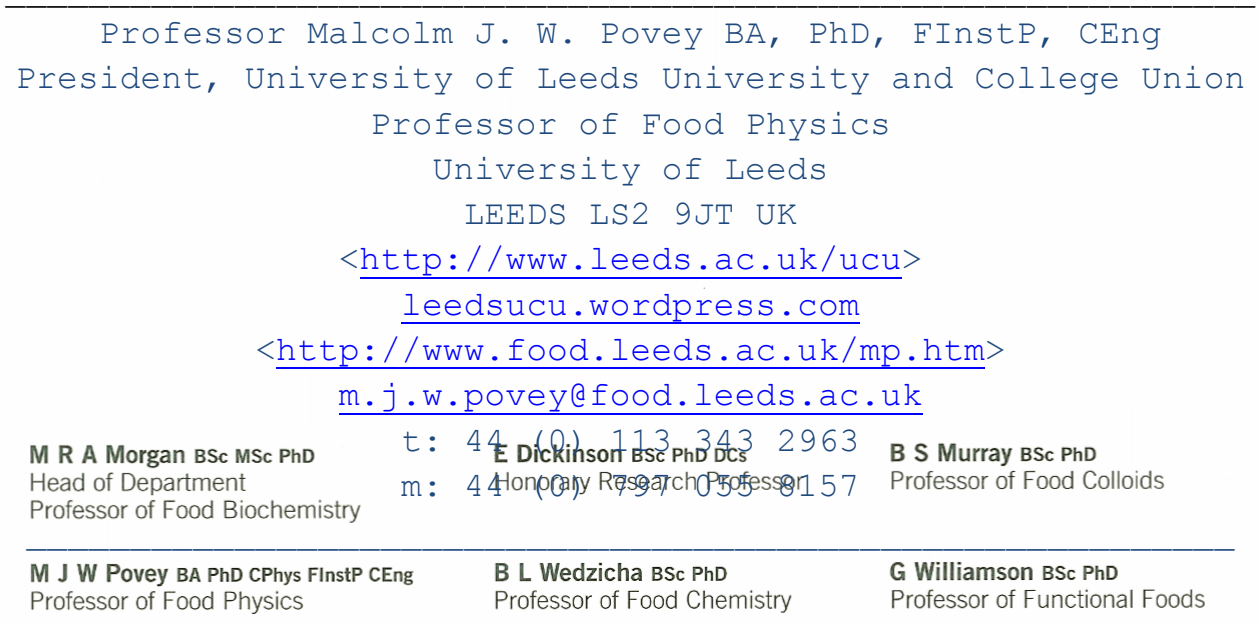


School of Food Science and Nutrition

The University of Leeds

Leeds LS2 9JT

TX 556473 UNILDS G

T +44 (0) 1132431751 (Direct Line)

T +44 (0) 1133432958 (General)

T +44 (0) 1133432959 (Head of Dept)

F $+44(0) 1133432982$

UNIVERSITY OF LEEDS

M R A Morgan BSc MSc PhD

Head of Department

Professor of Food Biochemistry

M J W Povey BA PhD CPhys FInstP CEng Professor of Food Physics
E Dickinson BSc PhD DCs

Honorary Research Professor

B L Wedzicha BSc PhD Professor of Food Chemistry
B S Murray BSc PhD

Professor of Food Colloids

G Williamson BSc PhD

Professor of Functional Foods 


\section{Author's reply to reviewers comments.}

We are very grateful to the reviewers for their careful reading of our manuscript and their valuable comments. Our replies (In blue italics) follow each comment below. We have provided a word manuscript using 'track changes' to mark our changes.

\section{Reviewers' comments:}

Reviewer \#1: In this work, the authors report ultrasonic properties of BSA solutions $(\mathrm{pH} 7)$ before and after various heating steps. They characterize the resultant aggregation be dynamic light scattering and chromatography and the properties of gels forms by small deformation rheological measurements. As the authors describe, various authors have used ultrasonic methods to characterize the effects of thermal treatments on proteins. My biggest difficulty in this work was deciphering the important and novel findings. In general a shorter and more focused paper would be helpful.

What is the major distinction between the current work and the Apenten paper by some of the main authors? Are there any numbers to compare between papers? In general wherever possible provide direct numerical comparisons with data from other studies. What types of figures have been reported for protein density, ultrasonic velocity and compressibility?

The major distinctions between the current work and the Apenten work are that we measured (a) over a wide frequency range $(2-160 \mathrm{MHz})$ rather than at one frequency $(2.25 \mathrm{MHz}) ;(b)$ isothermally rather than by scanning temperature at $20 \mathrm{C} \mathrm{hr}-1$; (c) both velocity and attenuation.

We have added a detailed comparison of data from our previous work and other studies, quoting data where available.(Table 2 and )

P14, last sentence: I am not clear why protein folding thermodynamics would lead to differences between the analytical methods. Also there really hasn't been much discussion of protein denaturation to this point.

We have omitted this since it overly complicates the argument.

P15, first sentence: I don't see anything in Figure 4 that could support a statement on the reproducibility of the experiment nor on the kinetics of aggregation. Further down that page the authors state that the ICHOS technique is sensitive to oligermerization. Is it not equally possible that the changes seen are due to denaturation?

We have added the standard error and the number of experimental replicates to Figure 4 to indicate the reproducibility of our experiments. No statements are made regarding the kinetics of aggregation, except to note that aggregation appears to be largely complete after $1 \mathrm{~h}$ heat treatment. This is based on the fact that little measureable change in SEC or ultrasound data can be seen after that time, up to a further two hours of measurement. This has been clarified in the paragraph. It is possible that denaturation contributes to the measured changes, however, there is 
no accompanying change in the velocity of sound which would be expected if denaturation was occurring.

Again on P14 Table 4 is referenced for the first time to state that the ICHOS method cannot distinguish the proportions of the oligomers. I don't follow what is being listed in the table, how the numbers were calculated, or how it supports the conclusion

We agree that the presence here of Table 4 is misleading. It is an estimate of the proportions of oligomers obtained for the experiment conducted at $70^{\circ} \mathrm{C}$ and is based solely on DLS data.

Overall the first paragraph on P15 took me a long time to read and understand and would probably beefit from a rewrite.

This paragraph has been rewritten.

I found Figure 9 hard to follow and unhelpful. Surely the position of the median size within each peak is less important than the population of the peak.

This Figure has been removed from the paper.

In the chromatography data, how were the peaks deconvoluted? Is it certain that the three peaks correspond to monomers, dimmers and trimers?

The column was calibrated using a set of protein standards covering a range of known molecular weights (Blue Dextran (2000 kDa), Thyroglobulin (Bovine) (669 kDa), IgG (Bovine) (150 kDa), Serum Albumin (Bovine) 66kDa, Ovalbumin (Chicken) 44 kDa, Lysozyme (Chicken) 14.5 kDa .

The procedure followed was a modification of the methods of Whitaker [19] and Andrews [20].

Whitaker, J.R., Determination of Molecular Weights of Proteins by Gel Filtration of Sephadex. Anal Chem, 1963. 35(12): p. 1950-3.

Andrews, P., Estimation of the molecular weights of proteins by Sephadex gel-filtration. Biochem J, 1964. 91(2): p. 222-33.

Void volume ( $\mathrm{Vo}$ ) was determined by recording the elution volume (retention time) of Blue Dextran at $280 \mathrm{~nm}$. The elution volume ( $\mathrm{Ve}$ ) of each protein in the Mw marker mixture was determined at $280 \mathrm{~nm}$ by observing the retention time recorded by the instrument software.

A standard curve was created by plotting the Log10 of the Mw vs. Ve/No for each protein in the mixture. Ve/No values were calculated for the unknown samples and the standard curve used to determine the estimated Mw.

This detail has been added to the manuscript

Was there any effort made to degas the gels and viscous solutions?

No, however, the headspace was degassed using steam during the canning process, and prior to heating.

I found Figure 10 unnecessary and the relevant observations could easily be conveyed in the text.

This Figure has been removed from the paper. 
P19, Does the Clark, Kavanaugh and Ross-Murphy paper state that linear fibril formation is a general phenomenon in protein gelation? (I don't have a copy in front of me but I don't remember that). Seems to contradict the Boye ref in the next paragraph.

Clark, Kavanagh and Ross-Murphy indeed say as quoted "network building appears to involve three main stages: initial protein unfolding, linear fibrillar aggregation, and random cross-linking of the fibrils" It does indeed appear to contradict Boye et al whose point of view is more consistent with our data. However, we were unable to make detailed measurements on the final stiff gel. We have added a comment to this effect.

The first complete sentence on P20 would seem to be a better fit with the start of the denaturation section before the discussion of aggregation mechanisms. The second complete sentence on that page introduces surface activity. This is the first mention of surface activity and appears to make no sense in context. The whole protein denaturation section seems to contain a lot of detail not really germane to the manuscript.

We have shortened the discussion according to this referees comments.

The first sentence on P21 seems to suggest that ultrasonic attenuation changes depends on free energy differences. Is ultrasonic velocity directly related to free energy?

Isothermal compressibility is given by the pressure gradient of the chemical potential which is related to the Gibbs Free Energy. The ultrasound velocity depends on the adiabatic compressibility which contains thermal terms as well and upon density. So it is not simply related to the free energy, although it can be argued that it is 'directly' related because there is a relationship. A comment along these lines has been incorporated into the paper.

In many cases the axis labels in the figures reproduces incorrectly.

The publisher's pdf converter inaccurately converts our word documents to pdf. We have attended to this problem.

Check the abbreviation for cooking time in Figure 11 and 12

Corrected to $\min$.

Is the oscillation in the G" data real (Figure 11).

The wiggles in the data are well within instrumental error. However, we do not have an explanation for these.

Reviewer \#2: The manuscript was presented in a clear and organised manner. However, there are several points that needed to be clarified.

General comments and corrections

1. Please number each line of the manuscript for the ease of reviewing

We have done this.

2. Please elaborate the novelty of present investigation, with the aid of suitable citations. It was not clearly reveal in the introduction sections. 
3. Please explain clearly the key advantages of using ultrasound spectroscopy in characterizing BSA denaturation. Indicate clearly in the introduction and / or results and discussion

4. Please explain the rationale and reason of not using circular dichroism (CD), and probably compared with the results of previous work with the current results, in the results and discussion

Points 2 to 4 above have been addressed in a modified introduction.

Specific corrections

1. Figure 1, 3, 7, 9, 11: Please provide a better quality figure to indicate the $y$-axis clearly.

This was caused by a fault in the word to pdf conversion during document submission and has now been corrected.

2. Section 2.4.2: Please explain the reason of using multiple wavelength detector in Size Exclusion Chromatography. Please provide valid citation, if this is a validated method. Usually, SEC was detected using RI or MALS detector.

\section{This has been done in the reply to the first referee.}

Reviewer \#3: The work by Povey et al. reported ultrasonic spectroscopic study on the denaturation and gelation of bovine serum albumin. The study provided a new insight into the structure change, molecular interaction, and molecule-solvent interaction during denaturation and gelation of proteins. The reviewer would recommend the publication of the paper after the following points have been considered during revision:

1) The structure of the paper can be better balanced. For example, the theoretical background and instrumentation of ultrasonic spectroscopy should be shortened, and only the essential equations necessary for the discussion of data presented. More significantly, the authors reviewed extensively and in details the previous works on contribution of molecular relaxation to ultrasonic attenuation. This was just for the purpose of demonstrating that even without separating out the relaxation contribution their data could be described by scattering model. Such a detailed review of molecular relaxation therefore seems not absolutely relevant, and can be briefed.

\section{The discussion has been extensively revised.}

2) In page 16, the authors stated G' and G" crossed at roughly two minutes. There however seemed to be no a real crossover between G' and G" from Figure 11; they just approached each other. Considering this and the DLS data that showed no large scale aggregation before 10 mins, I would prefer to think that gelation did not occurred until 10 mins. The author however argued that the gel started to form between 100 s and 300 s.

The text has been altered in accordance with the above comment.

3) Most of the equations were not displayed correctly in the pdf file.

This was due to a problem in the pdf converter which has been addressed. The original was a word document.

4) The axes and axis labels of most of the figures, particularly in Figure 1, 3, 7, 9 and 11, need to be made clear. 
This was also a problem caused by the pdf converter, which has been addressed.

5) In Figure 5, Please give the wavelength at which the absorption was measured.

The wavelength was $280 \mathrm{~nm}$, more detail is given in the reply to the first referee. 